\title{
Modelling the tensile failure of composites with the floating node method
}

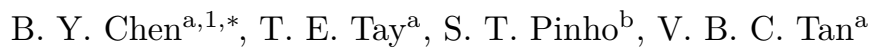 \\ ${ }^{a}$ Department of Mechanical Engineering, National University of Singapore. 21 Lower Kent Ridge Road, Singapore 119077 \\ ${ }^{b}$ Department of Aeronautics, South Kensington Campus, Imperial College London. London SW7 $2 A Z$, United Kingdom
}

\begin{abstract}
This paper presents the modelling of tensile failure of composites using novel enriched elements defined based on the floating node method. An enriched ply element is developed, such that a matrix crack can be modelled explicitly within its domain. An enriched cohesive element is developed to incorporate the boundaries of matrix cracks on the interface, such that the local stress concentrations on the interface can be captured. The edge status variable approach allows the automatic propagation of a large number of matrix cracks in the mesh. A laminate element is formed, such that a fixed, planar mesh can be used for laminates of arbitrary layups. The application examples demonstrate that the proposed method is capable of predicting several challenging scenarios of composites tensile failure, such as the large number matrix cracks, grip-to-grip longitudinal splits, widespread delamination, explosive splitting and distributed fibre breaking in the 0 plies, etc. The complete failure process of ply-blocked composite laminates, up to the final breaking of the loosened $0^{\circ}$ strips, are here firstly reproduced by modelling.
\end{abstract}

Keywords:

floating node method, composites, modelling, matrix crack, delamination, open-hole tension

\section{Introduction}

Fibre-reinforced composites have been widely used in the aerospace industry due to their properties such as high strength-to-weight ratio and high fatigue resistance, etc. The accurate prediction of the failure of composites is important for ensuring the safety and optimality of the design of composite structures. The progressive tensile failure of composites is characterised by the development of a large quantity of matrix cracks and delamination [1]. Matrix cracks in angle plies often trigger the onset of local delamination and migrate their way to the 0 plies, causing fibre breaking of the 0 plies or delamination on their surfaces [1-4]. When the laminate is of a ply-blocked configuration, i.e., plies of the same fibre angles are bundled together, longitudinal matrix splits tend to grow, sometimes from grip to grip. They cause widespread delamination, which subsequently lead to the disintegration of the laminate prior to the breaking of the stand-alone 0 ply-block $[1,2,4]$ (Figure 1). In fact, the breaking of the 0 ply-block itself is a challenging problem to be modelled. Fibre fracture rarely propagates along a well-defined path. On the contrary, it is often accompanied by an explosive occurrence of matrix splits, which separate the ply-block into loosened

\footnotetext{
* Corresponding author

Email address: boyang.chen.cn@gmail.com (B. Y. Chen)

${ }^{1}$ Tel: +65 90286157. Address: Block EA, \# 02-21, 9 Engineering Drive 1, Singapore 117575 


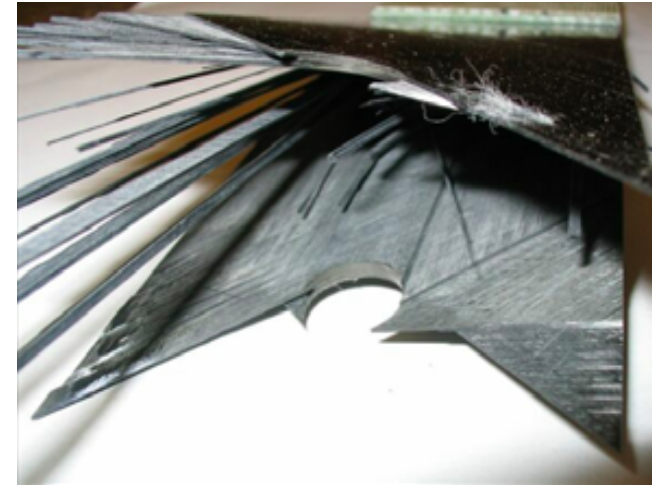

(a) Final failure image of the notched $\left[45_{4} / 90_{4} /-45_{4} / 0_{4}\right]_{\mathrm{S}}$ laminate in [2].

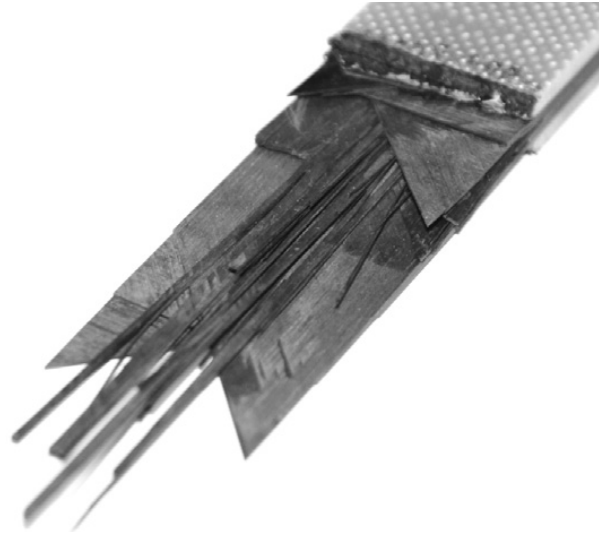

(b) Final failure image of the unnotched $\left[45_{4} / 90_{4} /-\right.$ $\left.45_{4} / 0_{4}\right]_{\mathrm{s}}$ laminate in $[1]$.

Figure 1: Typical tensile failure of ply-blocked laminates.

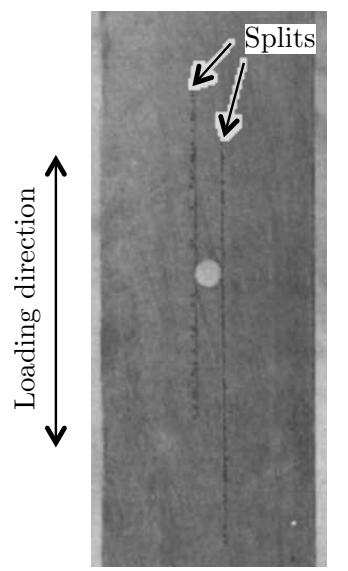

(a) Initial longitudinal splits

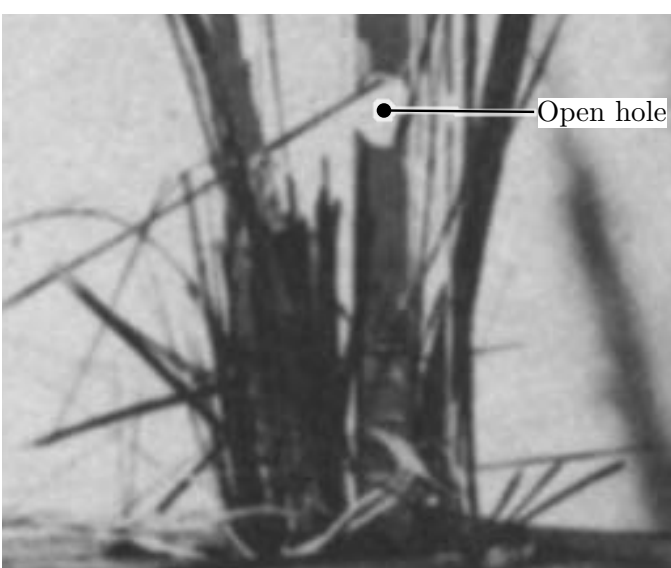

(b) Final failure scenario.

Figure 2: Typical tensile failure of a notched $0^{\circ}$ lamina [5].

strips, and the breaking of these individual strips causes the final load-drop [5] (Figure 2). These problems pose significant challenge for modelling, as a large quantity of different failure mechanisms, which often influence the development of each another, must be considered in the model. The faithful modelling of the above-mentioned scenarios are important for the accurate prediction of the final failure of composite laminates.

Different methods exist for the modelling of composites damage and failure. Cohesive elements are widely used when the potential fracture paths are known a priori, such as in the modelling of delamination [6-17]. While the paths of delamination are mostly the interfaces between different plies, the locations of matrix cracks are generally not known a priori. In the events where a stress concentrator dictates the failure process of the entire laminate, the mesh could be designed to incorporate this prior knowledge and cohesive elements or cohesive contact behaviour could be defined along the estimated paths of fracture [14, 18, 19]. For cases where this prior knowledge cannot be easily obtained, a more generic numerical method is needed to predict arbitrary matrix cracking. The smeared crack model (SCM) is developed such that a cohesive crack is 
"smeared" within the continuum element as diffused damage [20]. The SCM has been very successful for the modelling of many problems in the literature [20-24]. However, the SCM cannot enforce the direction of crack propagation $[18,25,26]$. In addition, the SCM models a sharp matrix crack with a band of softening elements [20] and the sharp crack boundaries are effectively approximated by blunt notches. The SCM is therefore unsuited when the local stress concentrations at the crack boundaries are the critical driving forces of progressive damage, such as in the case of delamination failure of ply-blocked laminates under tensile loading [1, 2, 4]. The eXtended Finite Element Method (XFEM) opens up the possibility of explicitly representing a crack within an element using additional DoF at the nodes and the Heaviside enrichment function for interpolation [27-36]. In the event of cracking, the displacement field in the element domain is interpolated by both its original shape functions and the Heaviside enrichment function (whose nodal values are represented by the extra DoF). The Hansbo's method [37], or otherwise known as the Phantom Node Method (PNM) [38-42] and the Augmented Finite Element Method (AFEM) [43-45], is an equivalent alternative to XFEM for the modelling of crack propagation within an element. It partitions the original element into two superposing Sub-Elements (SE), and each SE is integrated only in one of the crack-separated subdomains. These methods model the matrix cracks mesh-independently, and captures well the matrix crack/delamination interaction. However, interpolation and subdomain integration could introduce complexity and error in the calculation [46]. Complications could arise, if boundary conditions were to be applied on the crack surfaces [47, 48] (e.g., pressure loading in high-pressure environment, or displacement loading by cutting tools).

Despite the considerable efforts made toward the modelling of composites progressive failure, the high density of matrix cracks as seen in the experiment is still a challenging scenario to be matched by simulation. The full sequence of events in the progressive tensile failure of ply-blocked laminates [1, 2], i.e., the full-area delamination, grip-to-grip longitudinal splits and the final explosive fibre splitting and breaking of the almost independent 0 plies (Figure 1), remain to be fully captured by modelling. This paper presents the modelling of the above-mentioned phenomena with a three-dimensional (3D), composites-oriented development of the Floating Node Method (FNM) [46, 49]. The 3D FNM offers several capabilities:

- it can model a very high density of matrix cracks explicitly within the existing mesh;

- it can capture the local stress concentrations (caused by matrix cracks/splits from both the top and bottom plies) on the interfaces, and predict the thereby-induced local delamination;

- it can model the widespread, full-scale delamination;

- it can model the massive, grip-to-grip longitudinal splits in the 0 ply-block, together with the distributed fibre breaking in the loosened $0^{\circ}$ strips; and

- it allows the construction of a laminate element, where the layup is a defining parameter of the element. Consequently, a fixed, planar mesh can be used for arbitrary layups, thereby greatly simplifying the preprocessing.

The structure of the paper is organised as the following. The enriched definition of an element including edge connectivity and floating DoF is presented in Section 2. The details of the enriched elements for composites are presented in Section 3. The failure theories employed are described in Section 4. The application examples are shown in Section 5. Finally, the conclusions are drawn in Section 7. 


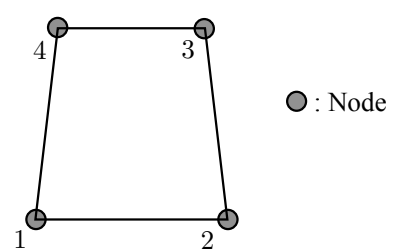

(a) Nodal definition of an element.

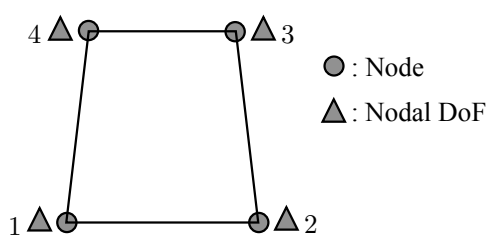

(c) DoF allocations of a standard element.

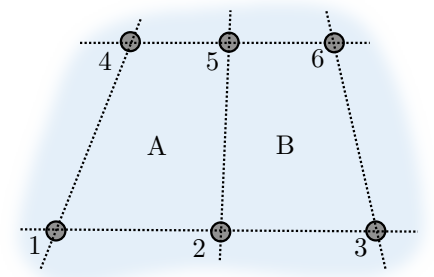

Element $A$

Nodes : $1,2,5,4$

Element B

Nodes : $2,3,6,5$

(b) A standard FE mesh and element connectivities.

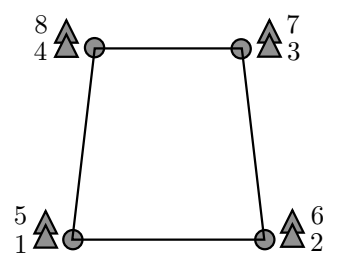

(d) DoF allocations of an XFEM (Heaviside enrichment) or PNM element.

Figure 3: Nodal definition of an element and nodal DoF allocations.

\section{Enriched element definition: edge connectivity and floating DoF}

A standard finite element is defined by its nodal connectivity (see Figure 3a and Figure 3b) and interpolation functions (i.e., shape functions). Although elements contain other shared geometrical entities such as edges and surfaces, these entities are not needed for the calculation in the standard FEM. Based on the assumption that the domain is a continuum throughout the analysis, only nodal values of the solution are required, and the solution over the rest of the domain is interpolated from the nodal values using shape functions. Therefore, in the standard FEM, DoFs are implicitly assumed to be nodal, i.e., they are always allocated to the nodes (Figure 3c). In the event of crack initiation and propagation, the original domain becomes discontinuous across the crack boundary, and the interpolation from nodal solutions using the continuous shape functions of the standard FEM fail to predict the solution jump across the crack. Additional sets of DoFs and interpolation functions are needed to represent the movement of the new crack boundaries. In XFEM, the nodal definition of an element has been followed. The additional DoF sets are allocated to the original nodes (Figure 3d). A Heaviside enrichment function is used for solution interpolation, and a subdomain scheme is used for integration (Figure 4).

The objective of modelling crack propagations without remeshing is to be able to represent a crack within existing elements. In this case, it is effectively the edges, surfaces, volumes, etc., which are split when cracks pass through the elements. These entities no longer remain continuous throughout the analysis, and additional DoFs are needed to represent the movements of the new, crack-induced boundaries on these entities. If it were possible to directly allocate DoFs to generic geometrical entities including edges, surfaces, volumes, etc., then the modelling of a crack within an element could be greatly simplified. The DoFs could be directly used to represent the displacements of the crack nodes on these entities. The key is that the DoFs must not be tied to a particular node a priori.

In this regard, the definition of an element is enriched in the FNM. In the 2D context, an element is defined with both a node connectivity and an edge connectivity, where the global indices of its nodes and 


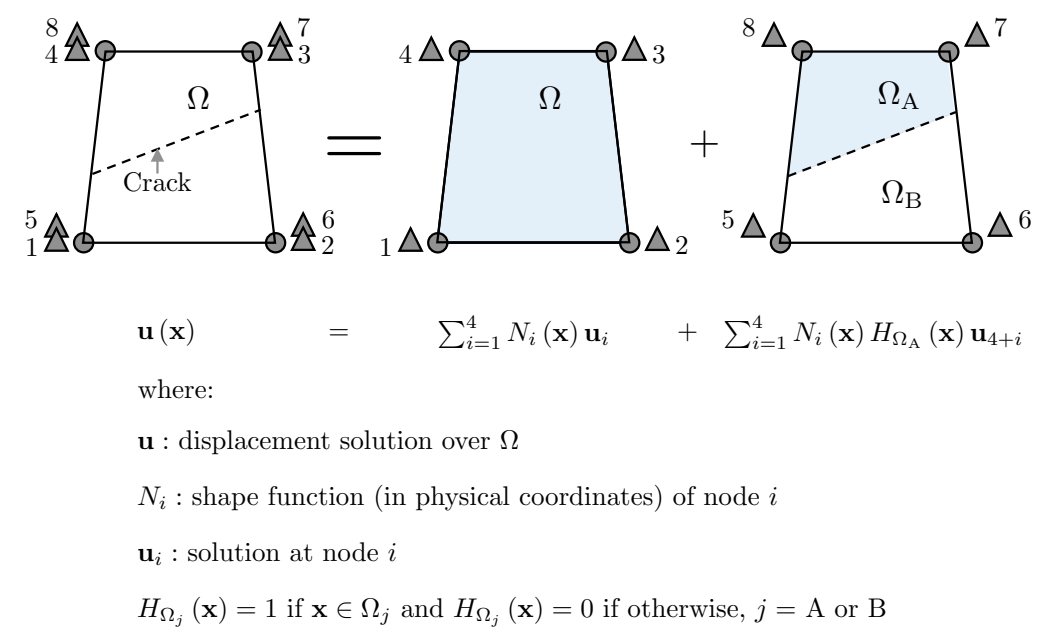

Figure 4: Modelling a crack with XFEM.

edges are recorded according to an ordered convention (Figure 5a). All the edges in the mesh are globally indexed (Figure 5b). In addition, the notion of floating DoF is proposed. In contrast to the nodal DoFs, a floating DoF is a DoF vector which is freely allocated to any geometrical entity. It can be used to represent the DoF of an arbitrary node on this entity, the location of which may not be known a priori. With the floating DoF and the enriched element definition, it is then possible to design an element with evolving topology $[46,49]$. If a pair of floating DoF sets is allocated to two edges of an element (Figure 5c), then the element is capable of modelling a crack within its domain (Figure 5d). The definitions of the element, i.e., the connectivities of its nodes, edges and DoF sets, remain unchanged throughout the analysis. More details of the 2D FNM elements for the modelling of matrix crack/delamination interaction problems can be found in $[46,49]$.

The FNM requires floating DoF sets to be allocated to element edges. However, in the existing commercial FEA packages (e.g., Abaqus), a DoF vector is implicitly assumed to be nodal. The straightforward allocation of DoFs to generic geometrical entities is not supported. In this context, a floating "node" is defined, such that its coordinates are random (e.g., the origin) and never used in the analysis. Its associated DoF set is allocated to an arbitrary geometrical entity (e.g., an edge), and is used to represent the displacement of another node on this entity, whose coordinates may not be known a priori.

The enriched definition of an element which includes edge connectivity and the notion of floating DoF enables the constructions of flexible elements whose behaviours can be tailored for specific applications. The following sections demonstrate the constructions of enriched composite ply, cohesive and laminate elements using the FNM, for the purpose of modelling matrix cracks, delamination and matrix crack/delamination intersections in the progressive tensile failure of composite laminates.

\section{3D Floating Node Method for composites}

\subsection{Enriched ply element}

An enriched ply element can be constructed with the FNM, such that a matrix crack can be modelled within its domain. Figure 6a shows the node, edge and DoF definitions of this element. It is assumed 


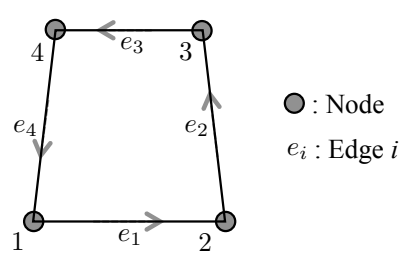

(a) Definition of an element in FNM: nodes and edges.

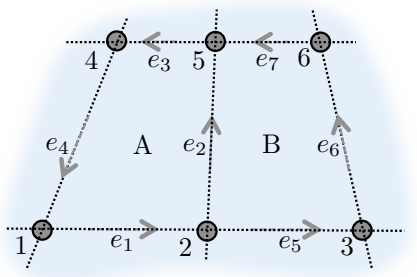

Element $\mathrm{A}$

Nodes : $1,2,5,4$

Edges : 1, 2, 3, 4

Element B

Nodes : $2,3,6,5$

Edges : $5,6,7,-2$

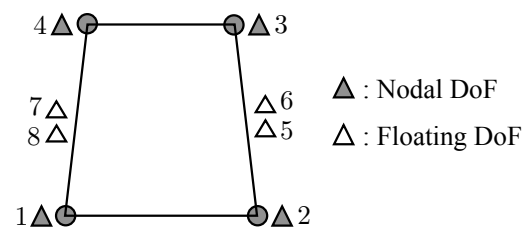

(c) Floating DoF sets can be allocated to edges of an element. (b) A FNM mesh and element connectivities.
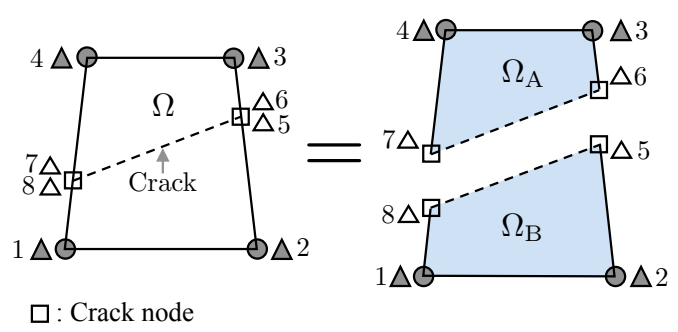

(d) Modelling of a crack using floating DoFs.

Figure 5: Edge connectivity and floating DoF, and their use on the modelling of a crack within an element.

that only the horizontal edges are crossed by the matrix crack. This is applicable when the matrix crack is perpendicular to the shell plane, which is typically the case in in-plane tensile loading situations. Under this assumption, floating DoF sets are only allocated to the horizontal edges Figure 6a.

\subsubsection{Before matrix cracking}

Before matrix cracking, the element is simply a standard linear brick element. The static equilibrium of a body with volume $\Omega$ under body forces with density $\mathbf{f}$ (acting on $\Omega$ ) and traction $\mathbf{t}$ acting on the boundary $\Gamma_{\mathrm{t}}$ can be expressed in the weak form as:

$$
\int_{\Omega} \boldsymbol{\epsilon}^{\mathrm{T}}(\mathbf{v}) \boldsymbol{\sigma}(\mathbf{u}) \mathrm{d} \Omega=\int_{\Omega} \mathbf{v}^{\mathrm{T}} \mathbf{f} \mathrm{d} \Omega+\int_{\Gamma_{\mathbf{t}}} \mathbf{v}^{\mathrm{T}} \mathbf{t} \mathrm{d} \Gamma
$$

where $\mathbf{u}$ is the displacement solution, $\mathbf{v}$ is the test function, $\mathbf{\epsilon}$ is the strain tensor (related to $\mathbf{u}$ through the differential operator relative to Cartesian coordinates $\mathcal{L}_{\mathbf{x}}$ as $\boldsymbol{\epsilon}=\mathcal{L}_{\mathbf{x}}(\mathbf{u})$ ), and $\boldsymbol{\sigma}$ is the stress tensor (related to the strains through Hooke's law as $\boldsymbol{\sigma}=\mathbf{D} \boldsymbol{\epsilon}$, with $\mathbf{D}$ being the constitutive tensor).

The nodal coordinates of the element are:

$$
\mathbf{x}_{\Omega}=\left[\mathbf{x}_{1}, \mathbf{x}_{2}, \ldots, \mathbf{x}_{8}\right]^{\mathrm{T}} .
$$

Its displacement solution at $\mathbf{x}$ is:

$$
\mathbf{u}(\mathbf{x})=\mathbf{N}(\mathbf{x}) \mathbf{u}_{\Omega},
$$

where $\mathbf{N}$ is the shape function matrix (in physical coordinates) of the nodes in $\mathbf{x}_{\Omega}$, and $\mathbf{u}_{\Omega}$ is the corre- 


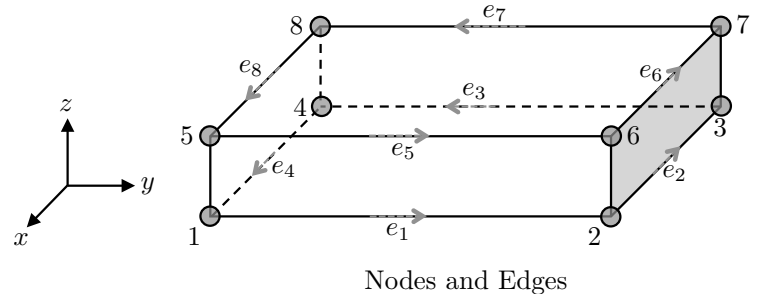

: Node

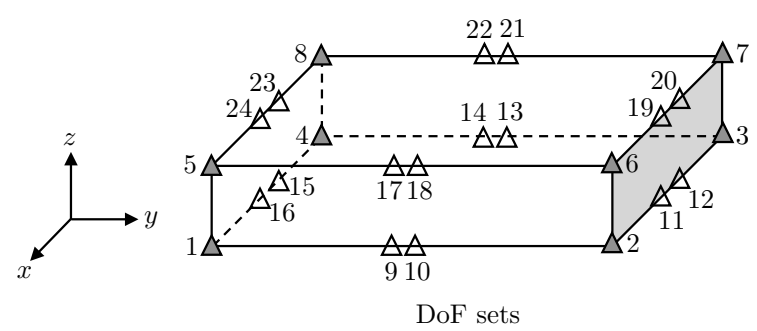

$e_{i}$ : Edge $i$

$\triangle:$ Nodal DoF

$\triangle$ : Floating DoF

(a) The definitions of an enriched ply element.

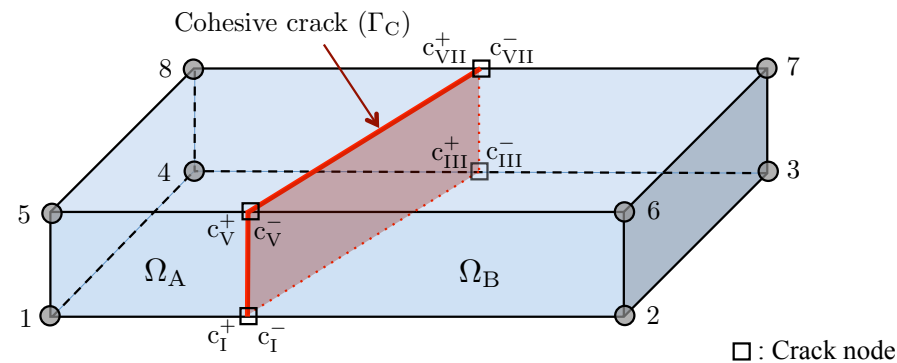

(b) A matrix crack introduces four pairs of coinciding crack nodes on four edges of the element

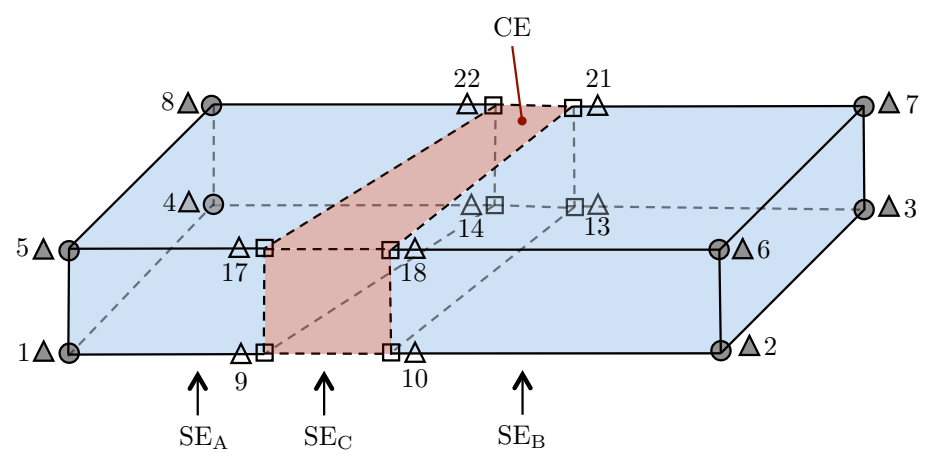

(c) Partition of SEs with respect to the matrix crack.

Figure 6: An enriched ply element by FNM. 
sponding nodal DoF vector. The strains can be expressed as:

$$
\boldsymbol{\epsilon}=\mathcal{L}_{\mathbf{x}}(\mathbf{u})=\mathbf{B} \mathbf{u}_{\Omega} \quad \text { with } \quad \mathbf{B}=\mathcal{L}_{\mathbf{x}}(\mathbf{N})
$$

leading to the stiffness matrix:

$$
\mathbf{K}_{\Omega}=\int_{\Omega} \mathbf{B}^{\mathrm{T}} \mathbf{D B} \mathrm{d} \Omega
$$

and to the vector of nodal forces:

$$
\mathbf{Q}_{\Omega}=\int_{\Omega} \mathbf{N}^{\mathrm{T}} \mathbf{f} \mathrm{d} \Omega+\int_{\Gamma_{\mathrm{t}}} \mathbf{N}^{\mathrm{T}} \mathbf{t} \mathrm{d} \Gamma
$$

The weak form of equilibrium equation, Equation 1, becomes then:

$$
\mathbf{K}_{\Omega} \mathbf{u}_{\Omega}=\mathbf{Q}_{\Omega}
$$

Note that the floating DoF sets of the element are not used in the analysis. A dummy stiffness, e.g, 1, can be assigned to their corresponding diagonal terms in the stiffness matrix of the element to facilitate convergence. Otherwise, the corresponding rows and columns of these unused DoFs can simply be removed from the global system equation.

\subsubsection{After matrix cracking}

If a certain failure criterion or propagation criterion is met such that a matrix crack initiates or propagates within the element domain and cuts across four edges of the element, for instance, Edge 1, Edge 3, Edge 5 and Edge 7 , then it creates four pairs of initially coinciding crack nodes, $c_{\mathrm{I}}^{ \pm}, c_{\mathrm{III}}^{ \pm}, c_{\mathrm{V}}^{ \pm}$and $c_{\mathrm{VII}}^{ \pm}$, on the four edges, respectively (Figure 6b). The coordinates of the crack nodes are fully defined by the intersections of the crack and the edges. Note that these crack nodes do not exist before the analysis, and hence no nodal DoFs could have been allocated to them a priori. In the FNM, the floating DoF sets allocated to the edges can be directly used to represent the displacements of the crack nodes on their respective edges (Figure 6c):

$$
\begin{gathered}
\mathbf{u}_{c_{\mathrm{I}}^{+}}=\mathbf{u}_{9}, \quad \mathbf{u}_{c_{\mathrm{I}}^{-}}=\mathbf{u}_{10}, \quad \mathbf{u}_{c_{\mathrm{III}}^{+}}=\mathbf{u}_{14}, \\
\mathbf{u}_{c_{\mathrm{V}}^{+}}=\mathbf{u}_{17}, \quad \mathbf{u}_{c_{\mathrm{V}}^{-}}=\mathbf{u}_{18}, \quad \mathbf{u}_{c_{\mathrm{VII}}^{+}}=\mathbf{u}_{22},
\end{gathered}
$$

where $\mathbf{u}_{c_{\mathrm{I}}^{ \pm}}, \mathbf{u}_{c_{\mathrm{III}}^{ \pm}}, \mathbf{u}_{c_{\mathrm{V}}^{ \pm}}$and $\mathbf{u}_{c_{\mathrm{VII}}^{ \pm}}$are the displacements of the crack nodes. The matrix crack partitions the original domain $\Omega$ into two subdomains, $\Omega_{\mathrm{A}}$ and $\Omega_{\mathrm{B}}$, with $\Gamma_{\mathrm{C}}$ being the crack interface. Assuming that the matrix crack is a cohesive crack, the weak form of the equilibrium equation now becomes:

$$
\int_{\Omega_{\mathrm{A}} \cup \Omega_{\mathrm{B}}} \boldsymbol{\epsilon}^{\mathrm{T}}(\mathbf{v}) \boldsymbol{\sigma}(\mathbf{u}) \mathrm{d} \Omega+\int_{\Gamma_{\mathrm{C}}} \llbracket \mathbf{v} \rrbracket^{\mathrm{T}} \boldsymbol{\tau}_{\mathrm{c}}(\llbracket \mathbf{u} \rrbracket) \mathrm{d} \Gamma=\int_{\Omega_{\mathrm{A}} \cup \Omega_{\mathrm{B}}} \mathbf{v}^{\mathrm{T}} \mathbf{f} \mathrm{d} \Omega+\int_{\Gamma_{\mathbf{t}}} \mathbf{v}^{\mathrm{T}} \mathbf{t} \mathrm{d} \Gamma
$$

where $\llbracket \bullet$ represents the jump of a function between the top and bottom surfaces of the cohesive crack, and $\boldsymbol{\tau}_{\mathrm{c}}$ is the traction acting between the surfaces of the cohesive crack. $\boldsymbol{\tau}_{\mathrm{c}}$ relates to the separation of the top and bottom surfaces of the cohesive crack, $\llbracket \mathbf{u} \rrbracket$, through a constitutive relationship of the form:

$$
\boldsymbol{\tau}_{\mathrm{c}}=\mathbf{D}_{\mathrm{CE}} \llbracket \mathbf{u} \rrbracket
$$


where $\mathbf{D}_{\mathrm{CE}}$ embodies the cohesive constitutive law.

Three SEs can be formed, such that they naturally represent the two bulk subdomains $\Omega_{\mathrm{A}}$ and $\Omega_{\mathrm{B}}$ and the cohesive crack $\Gamma_{\mathrm{C}}$ (Figure 6c). The nodal coordinates of the three SEs are:

$$
\begin{aligned}
& \mathbf{x}_{\Omega_{\mathrm{A}}}=\left[\mathbf{x}_{1}, \mathbf{x}_{c_{\mathrm{I}}^{+}}, \mathbf{x}_{c_{\mathrm{III}}^{+}}, \mathbf{x}_{4}, \mathbf{x}_{5}, \mathbf{x}_{c_{\mathrm{V}}^{+}}, \mathbf{x}_{c_{\mathrm{VII}}^{+}}, \mathbf{x}_{8}\right]^{\mathrm{T}}, \\
& \mathbf{x}_{\Omega_{\mathrm{B}}}=\left[\mathbf{x}_{c_{\mathrm{I}}^{-}}, \mathbf{x}_{2}, \mathbf{x}_{3}, \mathbf{x}_{c_{\mathrm{III}}^{-}}, \mathbf{x}_{c_{\mathrm{V}}^{-}}, \mathbf{x}_{6}, \mathbf{x}_{7}, \mathbf{x}_{c_{\mathrm{VII}}^{-}}\right]^{\mathrm{T}}, \\
& \mathbf{x}_{\Gamma_{\mathrm{C}}}=\left[\mathbf{x}_{c_{\mathrm{I}}^{-}}, \mathbf{x}_{c_{\mathrm{V}}^{-}}, \mathbf{x}_{c_{\mathrm{VII}}^{-}}, \mathbf{x}_{c_{\mathrm{III}}^{-}}, \mathbf{x}_{c_{\mathrm{I}}^{+}}, \mathbf{x}_{c_{\mathrm{V}}^{+}}, \mathbf{x}_{c_{\mathrm{VII}}^{+}}, \mathbf{x}_{c_{\mathrm{III}}^{+}}\right]^{\mathrm{T}} .
\end{aligned}
$$

With the floating DoF sets allocated to represent the displacements of the crack nodes (ref. Equation (8)), the DoFs of the nodes of the three SEs are fully defined:

$$
\begin{aligned}
& \mathbf{u}_{\Omega_{\mathrm{A}}}=\left[\mathbf{u}_{1}, \mathbf{u}_{9}, \mathbf{u}_{14}, \mathbf{u}_{4}, \mathbf{u}_{5}, \mathbf{u}_{17}, \mathbf{u}_{22}, \mathbf{u}_{8}\right]^{\mathrm{T}}, \\
& \mathbf{u}_{\Omega_{\mathrm{B}}}=\left[\mathbf{u}_{10}, \mathbf{u}_{2}, \mathbf{u}_{3}, \mathbf{u}_{13}, \mathbf{u}_{18}, \mathbf{u}_{6}, \mathbf{u}_{7}, \mathbf{u}_{21}\right]^{\mathrm{T}}, \\
& \mathbf{u}_{\Gamma_{\mathrm{C}}}=\left[\mathbf{u}_{10}, \mathbf{u}_{18}, \mathbf{u}_{21}, \mathbf{u}_{13}, \mathbf{u}_{9}, \mathbf{u}_{17}, \mathbf{u}_{22}, \mathbf{u}_{14}\right]^{\mathrm{T}} .
\end{aligned}
$$

Under the assumption of isoparametric representation, the displacement solution is:

$$
\begin{aligned}
\mathbf{u}(\mathbf{x}) & =\mathbf{N}_{\Omega_{\mathrm{A}}}(\mathbf{x}) \mathbf{u}_{\Omega_{\mathrm{A}}}, \text { if } \mathbf{x} \in \Omega_{\mathrm{A}} \\
& =\mathbf{N}_{\Omega_{\mathrm{B}}}(\mathbf{x}) \mathbf{u}_{\Omega_{\mathrm{B}}}, \text { if } \mathbf{x} \in \Omega_{\mathrm{B}},
\end{aligned}
$$

with

$$
\llbracket \mathbf{u} \rrbracket(\mathbf{x})=\mathbf{N}_{\Gamma_{\mathrm{C}}}(\mathbf{x}) \mathbf{u}_{\Gamma_{\mathrm{C}}}, \text { if } \mathbf{x} \in \Gamma_{\mathrm{C}},
$$

where $\mathbf{N}_{\Omega_{\mathrm{A}}}, \mathbf{N}_{\Omega_{\mathrm{B}}}$ and $\mathbf{N}_{\Gamma_{\mathrm{C}}}$ are the standard finite element shape function matrices (in physical coordinates) of the elements defined by $\mathbf{x}_{\Omega_{\mathrm{A}}}, \mathbf{x}_{\Omega_{\mathrm{B}}}$ and $\mathbf{x}_{\Gamma_{\mathrm{C}}}$, respectively. Note that the cohesive $\mathrm{SE}$ is entirely formed by the crack nodes and the floating DoF sets. The stiffness matrices of the SEs are (ref. Equation (5))

$$
\begin{aligned}
\mathbf{K}_{\Omega_{\mathrm{A}}} & =\int_{\Omega_{\mathrm{A}}} \mathbf{B}_{\mathrm{A}}^{\mathrm{T}} \mathbf{D} \mathbf{B}_{\mathrm{A}} \mathrm{d} \Omega, \\
\mathbf{K}_{\Omega_{\mathrm{B}}} & =\int_{\Omega_{\mathrm{B}}} \mathbf{B}_{\mathrm{B}}^{\mathrm{T}} \mathbf{D} \mathbf{B}_{\mathrm{B}} \mathrm{d} \Omega, \\
\mathbf{K}_{\Gamma_{\mathrm{C}}} & =\int_{\Gamma_{\mathrm{C}}} \mathbf{N}_{\Gamma_{\mathrm{C}}}^{\mathrm{T}} \mathbf{D}_{\mathrm{CE}} \mathbf{N}_{\Gamma_{\mathrm{C}}} \mathrm{d} \Gamma,
\end{aligned}
$$

where

$$
\begin{aligned}
& \mathbf{B}_{\mathrm{A}}=\mathcal{L}_{\mathbf{x}}\left(\mathbf{N}_{\Omega_{\mathrm{A}}}\right), \\
& \mathbf{B}_{\mathrm{B}}=\mathcal{L}_{\mathbf{x}}\left(\mathbf{N}_{\Omega_{\mathrm{B}}}\right) .
\end{aligned}
$$


Similarly, the force vectors of the two bulk SEs are (ref. Equation (6)):

$$
\begin{aligned}
& \mathbf{Q}_{\Omega_{\mathrm{A}}}=\int_{\Omega_{\mathrm{A}}} \mathbf{N}_{\Omega_{\mathrm{A}}}^{\mathrm{T}} \mathbf{f} \mathrm{d} \Omega+\int_{\Gamma_{\mathrm{t}} \cap \Gamma_{\Omega_{\mathrm{a}}}} \mathbf{N}_{\Omega_{\mathrm{A}}}^{\mathrm{T}} \mathbf{t} \mathrm{d} \Gamma, \\
& \mathbf{Q}_{\Omega_{\mathrm{B}}}=\int_{\Omega_{\mathrm{B}}} \mathbf{N}_{\Omega_{\mathrm{B}}}^{\mathrm{T}} \mathbf{f} \mathrm{d} \Omega+\int_{\Gamma_{\mathrm{t}} \cap \Gamma_{\Omega_{\mathrm{b}}}} \mathbf{N}_{\Omega_{\mathrm{B}}}^{\mathrm{T}} \mathbf{t} \mathrm{d} \Gamma,
\end{aligned}
$$

where $\Gamma_{\Omega_{\mathrm{a}}}$ and $\Gamma_{\Omega_{\mathrm{b}}}$ are the boundaries of $\Omega_{\mathrm{A}}$ and $\Omega_{\mathrm{B}}$, respectively. If no external loading is applied on the crack surfaces, the force vector of the cohesive $\mathrm{SE}, \mathbf{Q}_{\Gamma_{\mathrm{C}}}$, is simply a zero vector:

$$
\mathrm{Q}_{\Gamma_{\mathrm{C}}}=\mathbf{0} .
$$

The weak form of the equilibrium equation, Equation (9), can be written in an assembled form as:

$$
\mathbf{K}_{\mathrm{el}} \mathbf{u}_{\mathrm{el}}=\mathbf{Q}_{\mathrm{el}}
$$

with:

$$
\begin{aligned}
\mathbf{K}_{\mathrm{el}} & =\mathcal{A}\left(\mathbf{K}_{\Omega_{\mathrm{A}}}, \mathbf{K}_{\Omega_{\mathrm{B}}}, \mathbf{K}_{\Gamma_{\mathrm{C}}}\right), \\
\mathbf{u}_{\mathrm{el}} & =\mathcal{A}\left(\mathbf{u}_{\Omega_{\mathrm{A}}}, \mathbf{u}_{\Omega_{\mathrm{B}}}, \mathbf{u}_{\Gamma_{\mathrm{C}}}\right), \\
\mathbf{Q}_{\mathrm{el}} & =\mathcal{A}\left(\mathbf{Q}_{\Omega_{\mathrm{A}}}, \mathbf{Q}_{\Omega_{\mathrm{B}}}, \mathbf{Q}_{\Gamma_{\mathrm{C}}}\right),
\end{aligned}
$$

where $\mathcal{A}$ is the assembly operator. The diagonal stiffness terms corresponding to the unused floating DoF sets can be assigned dummy positive values to facilitate convergence. Otherwise, the rows and columns corresponding to the unused DoFs can be removed from the global equation before the solution process. Note that since the DoFs of a crack node are directly represented by a floating DoF set and are not interpolated from those of multiple nodes (as in the case of XFEM or PNM), the imposition of boundary conditions on the crack nodes (e.g., pressure loading in a high-pressure environment or displacement loading by cutting tools) are straightforward. It suffices to simply apply the equivalent nodal forces or displacement boundary conditions directly onto the floating DoFs of the crack nodes. The complications of this issue in XFEM $[47,48]$ is hence avoided.

The above example demonstrates the situation where the matrix crack partitions the original domain into two brick sub-domains. The same procedure applies to the case where the crack partitions the domain into a triangular prism and a pentagonal prism (Figure 7). It suffices to form five SEs using the original nodes and crack nodes, where the pentagonal prism is represented by three triangular prism SEs (Figure 7).

\subsubsection{Modelling of fibre failure}

Fibre failure usually marks the final failure of composites. In this work, the fibre failure is modelled in the ply elements and SEs using the smeared crack approach in [22], where the translaminar fracture toughness, $G_{\mathrm{fc}}$, scales with respect to ply-blocking:

$$
G_{\mathrm{fc}}^{n}=n \times G_{\mathrm{fc}}^{1} .
$$

$G_{\mathrm{fc}}^{1}$ is the toughness for a single ply, and $G_{\mathrm{fc}}^{n}$ is that for a ply-block of $n$ plies. This scaling is based on the experimental finding in [50], where the thicker ply-blocks had more fibre pull-out during fracture, leading to 

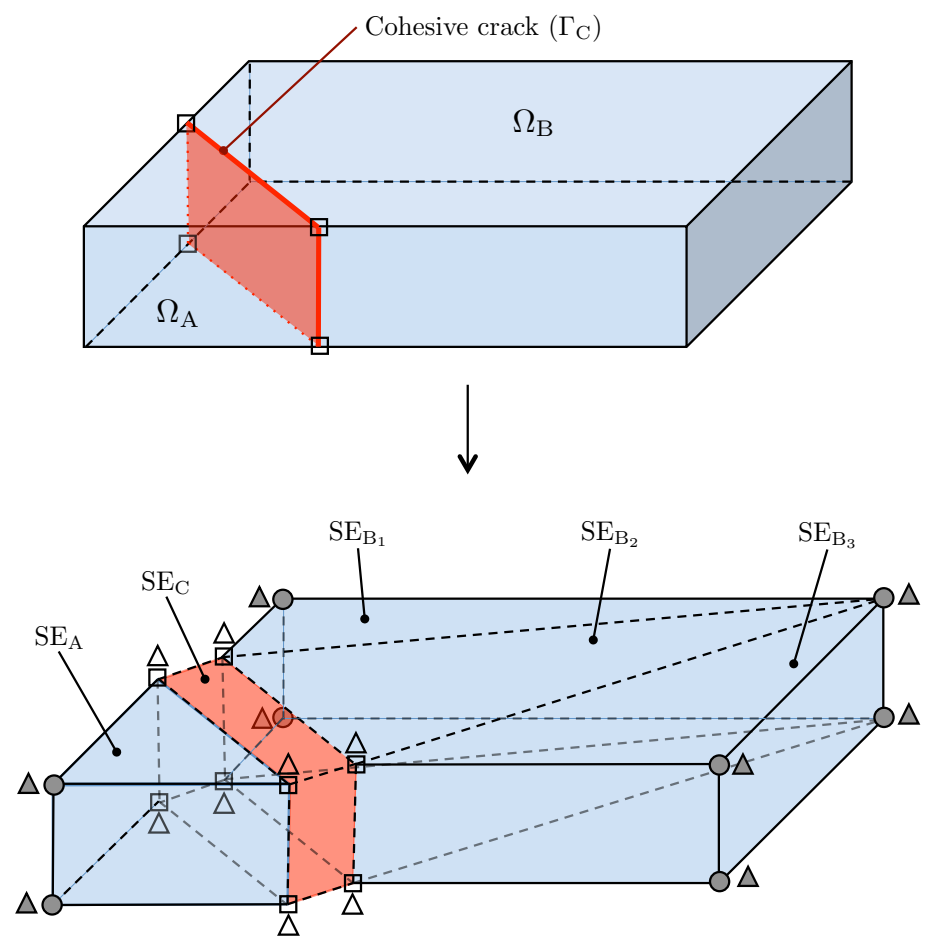

Figure 7: The ply element may also partition into four triangular prism SEs and a cohesive SE with respect to a matrix crack.

higher energy dissipations per unit area of projected crack propagation. The smeared crack model updates damage variables at the element integration points, and does not require modifications of the element topology or the integration scheme.

\subsection{Enriched cohesive element}

It has been shown in the literature that the explicit modelling of matrix crack boundaries on the interfaces is important for the accuracy in the prediction of matrix crack density and crack-induced delamination $[45,46]$ (see also Figure 8). In this section, an enriched 3D cohesive element is developed, such that the matrix crack boundaries are explicitly represented on the interface.

Figure 9a shows the definitions of the element. It consists of two initially coinciding surfaces, defined by both its nodes and its edges. A pair of floating DoF sets are allocated to each edge of the element.

Before any matrix crack appearing from the top or bottom ply element, the cohesive element is a standard 8-node linear cohesive element defined by the first 8 nodes of the element:

$$
\mathbf{x}_{\mathrm{CE}}=\left[\mathbf{x}_{1}, \mathbf{x}_{2}, \ldots, \mathbf{x}_{8}\right]^{\mathrm{T}}
$$

Only the nodal DoF sets, $\mathbf{u}_{\mathrm{CE}}$, are actually used in the calculation. The displacement jump at a point $\mathbf{x}$ of the cohesive interface is:

$$
\llbracket \mathbf{u} \rrbracket(\mathbf{x})=\mathbf{N}_{\mathrm{CE}}(\mathbf{x}) \mathbf{u}_{\mathrm{CE}}
$$

where $\mathbf{N}_{\mathrm{CE}}$ is the shape function matrix of the element. The stiffness matrix of the element is (see also $\mathbf{K}_{\Gamma_{\mathrm{C}}}$ 


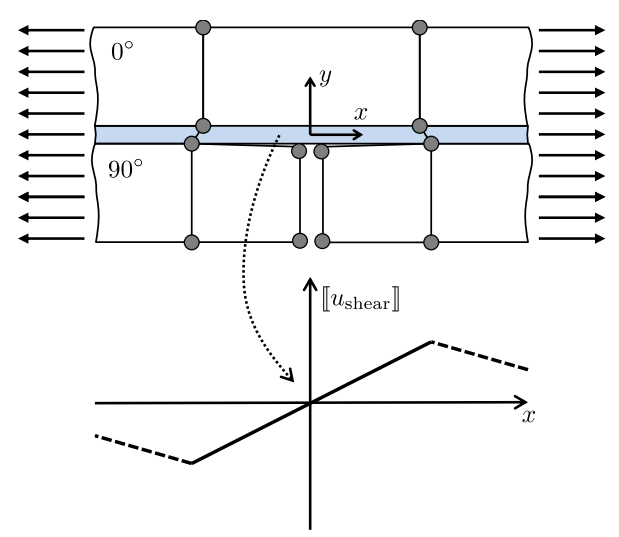

(a) A non-matching mesh at the matrix crack/interface intersection $[45,46]$.

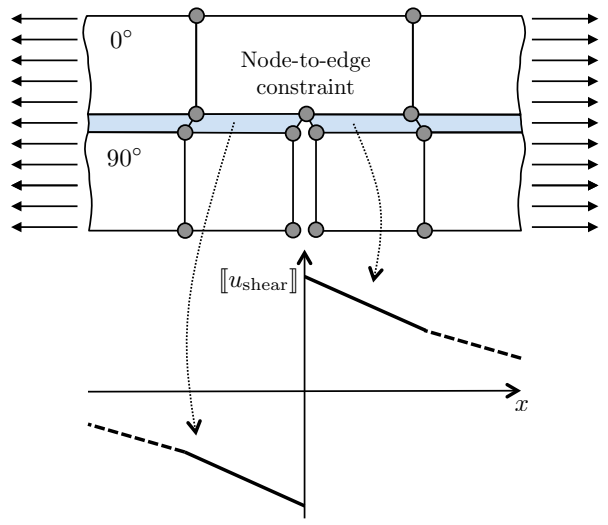

(b) A matching mesh representing the matrix crack/interface intersection $[45,46]$

Figure 8: Approximation of the shear displacement jump, $\llbracket u_{\text {shear }} \rrbracket$, across the $0 / 90$ interface. Non-matching mesh fails to capture the discontinuity in $\llbracket u_{\text {shear }} \rrbracket$ across the matrix crack boundary on the interface (a); matching mesh captures the discontinuity in $\llbracket u_{\text {shear }} \rrbracket$ across the crack boundary (b).

in Equation (15)):

$$
\mathbf{K}_{\mathrm{CE}}=\int_{\Gamma_{\mathrm{CE}}} \mathbf{N}_{\mathrm{CE}}^{\mathrm{T}} \mathbf{D}_{\mathrm{CE}} \mathbf{N}_{\mathrm{CE}} \mathrm{d} \Gamma
$$

where $\Gamma_{\mathrm{CE}}$ is the domain of the cohesive element. Similarly as in the case of the ply element, the unused DoF sets can be allocated dummy positive diagonal stiffness terms, or they can be removed from the final system equation.

If a matrix crack occurs on the top ply element and, for instance, cuts across Edge 5 and Edge 7 on the top surface of the cohesive element (Figure 9b), then it creates two pairs of initially coinciding crack nodes on these edges, i.e., $c_{\mathrm{V}}^{ \pm}$and $c_{\mathrm{VII}}^{ \pm}$, respectively. Two auxiliary nodes which initially coincide with the crack nodes can be located on the bottom surface of the cohesive element, ie., $c_{\text {I }}$ and $c_{\text {III }}$. These nodes allow the nodal coordinates of the two SEs, namely $\mathrm{SE}_{1}^{\mathrm{Top}}$ and $\mathrm{SE}_{2}^{\mathrm{Top}}$ (see Figure 9c), to be defined:

$$
\begin{aligned}
& \mathbf{x}_{\mathrm{SE}_{1}^{\text {Top }}}=\left[\mathbf{x}_{1}, \mathbf{x}_{c_{\mathrm{I}}}, \mathbf{x}_{c_{\text {III }}}, \mathbf{x}_{4}, \mathbf{x}_{5}, \mathbf{x}_{c_{\mathrm{V}}^{+}}, \mathbf{x}_{c_{\mathrm{VII}}^{+}}, \mathbf{x}_{8}\right]^{\mathrm{T}}, \\
& \mathbf{x}_{\mathrm{SE}_{2}^{\text {Top }}}=\left[\mathbf{x}_{c_{\mathrm{I}}}, \mathbf{x}_{2}, \mathbf{x}_{3}, \mathbf{x}_{c_{\mathrm{III}}}, \mathbf{x}_{c_{\mathrm{V}}^{-}}, \mathbf{x}_{6}, \mathbf{x}_{7}, \mathbf{x}_{c_{\mathrm{VII}}^{-}}\right]^{\mathrm{T}} .
\end{aligned}
$$

The floating DoF sets allocated to Edge 5 and Edge 7 can be used to directly represent the displacements of the crack nodes, hence:

$$
\mathbf{u}_{c_{\mathrm{V}}^{+}}=\mathbf{u}_{17}, \quad \mathbf{u}_{c_{\mathrm{V}}^{-}}=\mathbf{u}_{18}, \quad \mathbf{u}_{c_{\mathrm{VII}}^{+}}=\mathbf{u}_{22}, \quad \mathbf{u}_{c_{\mathrm{VII}}^{-}}=\mathbf{u}_{21} .
$$

The displacements of the two auxiliary nodes are represented by two auxiliary DoF sets, $\mathbf{u}_{25}$ and $\mathbf{u}_{26}$ :

$$
\mathbf{u}_{c_{\mathrm{I}}}=\mathbf{u}_{25}, \quad \mathbf{u}_{c_{\mathrm{III}}}=\mathbf{u}_{26}
$$

$\mathbf{u}_{25}$ and $\mathbf{u}_{26}$ are not independent DoF sets, as they represent the displacements of nodes on intact edges, where the displacement solution is fully characterised by the DoF values of the end nodes through shape 

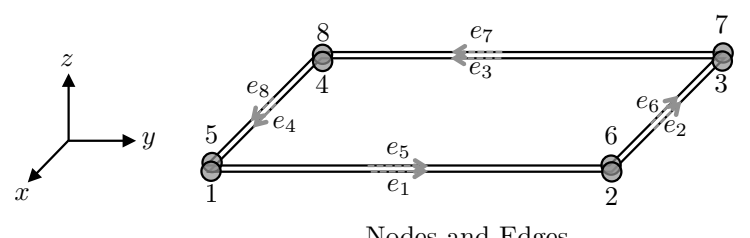

O : Node

Nodes and Edges

$e_{i}$ : Edge $i$

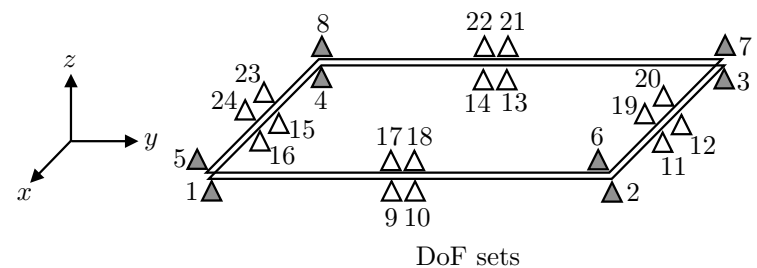

$\triangle$ : Nodal DoF

$\triangle$ : Floating DoF

(a) The definition of an enriched cohesive element.

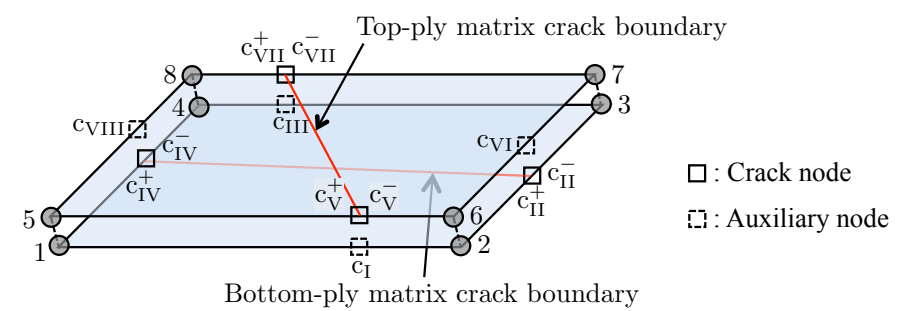

(b) Matrix cracks may occur in top and/or bottom plies.

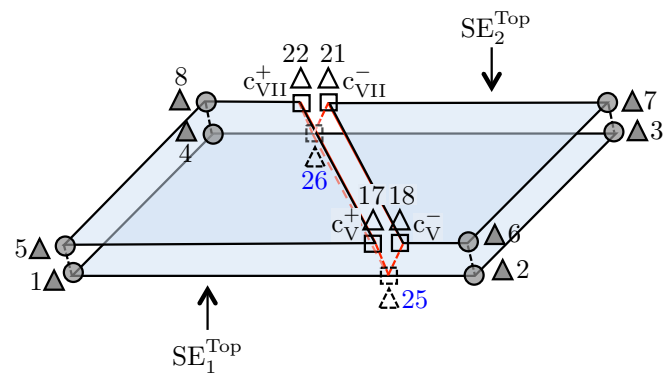

A : Auxiliary DoF

(c) $\mathrm{SE}^{\mathrm{Top}}$ and its $\mathrm{SEs}, \mathrm{SE}_{1}^{\mathrm{Top}}$ and $\mathrm{SE}_{2}^{\mathrm{Top}}$, with respect to the matrix crack boundary on the top surface.

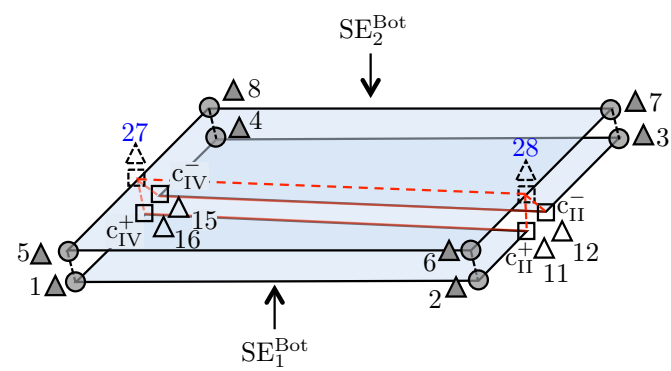

(d) $\mathrm{SE}^{\mathrm{Bot}}$ and its $\mathrm{SEs}, \mathrm{SE}_{1}^{\mathrm{Bot}}$ and $\mathrm{SE}_{2}^{\mathrm{Bot}}$, with respect to the matrix crack boundary on the bottom surface.

Figure 9: An enriched cohesive element by the FNM. 
function interpolation. Therefore:

$$
\begin{aligned}
& \mathbf{u}_{25}=N_{1}\left(\mathbf{x}_{c_{\mathrm{I}}}\right) \mathbf{u}_{1}+N_{2}\left(\mathbf{x}_{c_{\mathrm{I}}}\right) \mathbf{u}_{2}, \\
& \mathbf{u}_{26}=N_{3}\left(\mathbf{x}_{c_{\text {III }}}\right) \mathbf{u}_{3}+N_{4}\left(\mathbf{x}_{c_{\text {III }}}\right) \mathbf{u}_{4},
\end{aligned}
$$

where $N_{i}$ is the shape function of Node $i$.

The DoF vectors of the two SEs can be formed:

$$
\begin{aligned}
& \mathbf{u}_{\mathrm{SE}_{1}^{\mathrm{Top}}}=\left\{\mathbf{u}_{1}, \mathbf{u}_{25}, \mathbf{u}_{26}, \mathbf{u}_{4}, \mathbf{u}_{5}, \mathbf{u}_{17}, \mathbf{u}_{22}, \mathbf{u}_{8}\right\}^{\mathrm{T}} \\
& \mathbf{u}_{\mathrm{SE}_{2}^{\mathrm{Top}}}=\left\{\mathbf{u}_{25}, \mathbf{u}_{2}, \mathbf{u}_{3}, \mathbf{u}_{26}, \mathbf{u}_{18}, \mathbf{u}_{6}, \mathbf{u}_{7}, \mathbf{u}_{21}\right\}^{\mathrm{T}}
\end{aligned}
$$

The displacement jumps are:

$$
\begin{aligned}
& \llbracket \mathbf{u} \rrbracket(\mathbf{x})=\mathbf{N}_{\mathrm{SE}_{1}^{\mathrm{Top}}}(\mathbf{x}) \mathbf{u}_{\mathrm{SE}_{1}^{\mathrm{Top}}} \text {, if } \mathbf{x} \in \Omega_{\mathrm{SE}_{1}^{\mathrm{Top}}}, \\
& \llbracket \mathbf{u} \rrbracket(\mathbf{x})=\mathbf{N}_{\mathrm{SE}_{2}^{\mathrm{Top}}}(\mathbf{x}) \mathbf{u}_{\mathrm{SE}_{2}^{\mathrm{Top}}} \text {, if } \mathbf{x} \in \Omega_{\mathrm{SE}_{2}^{\mathrm{Top}}}
\end{aligned}
$$

where $\mathbf{N}_{\mathrm{SE}_{1}^{\text {Top }}}$ and $\mathbf{N}_{\mathrm{SE}_{2}^{\text {Top }}}$ are shape function matrices (in physical coordinates) of the nodes in $\mathbf{x}_{\mathrm{SE}_{1}^{\mathrm{Top}}}$ and $\mathbf{x}_{\mathrm{SE}_{2}^{\text {Top }}}$, respectively. The two SEs are fully defined. Their stiffness matrices are:

$$
\begin{aligned}
\mathbf{K}_{\mathrm{SE}_{1}^{\mathrm{Top}}} & =\int_{\Gamma_{\mathrm{SE}_{1}^{\mathrm{Top}}}} \mathbf{N}_{\mathrm{SE}_{1}^{\mathrm{Top}}}^{\mathrm{T}} \mathbf{D}_{\mathrm{CE}} \mathbf{N}_{\mathrm{SE}_{1}^{\mathrm{Top}} \mathrm{d} \Gamma,} \\
\mathbf{K}_{\mathrm{SE}_{2}^{\mathrm{Top}}} & =\int_{\Gamma_{\mathrm{SE}_{2}^{\mathrm{Top}}}} \mathbf{N}_{\mathrm{SE}_{2}^{\mathrm{Top}}}^{\mathrm{Top}} \mathbf{D}_{\mathrm{CE}} \mathbf{N}_{\mathrm{SE}_{2}^{\mathrm{Top}}} \mathrm{d} \Gamma,
\end{aligned}
$$

The above stiffness matrices can be assembled according to the DoF connectivities of the two SEs in Equation (29) to result in the stiffness matrix of $\mathrm{SE}^{\text {Top }}$ in Figure 9c:

$$
\mathbf{K}_{\mathrm{SE}}{ }^{\text {Top }}=\mathcal{A}\left(\mathbf{K}_{\mathrm{SE}_{1}^{\mathrm{Top}}}, \quad \mathbf{K}_{\mathrm{SE}_{2}^{\mathrm{Top}}}\right)
$$

The terms corresponding to the auxiliary DoF sets can be removed using the constraint equations in Equation (28). The Newton-Cotes scheme is used for the integration of cohesive elements or SEs. If damage has already initiated in the original cohesive element before the partition, then the damage variables are mapped onto the integration points of the SEs at the moment of partition.

Similarly, if a matrix crack occurs on the bottom ply element and, for instance, cuts across Edge 2 and Edge 4 of the bottom surface of the cohesive element, the $\mathrm{SEs}, \mathrm{SE}_{1}^{\mathrm{Bot}}$ and $\mathrm{SE}_{2}^{\mathrm{Bot}}$, can be formed by using the same procedure as described above. The stiffness matrices of the $\mathrm{SEs}, \mathbf{K}_{\mathrm{SE}_{1}^{\mathrm{Bot}}}$ and $\mathbf{K}_{\mathrm{SE}_{2}^{\text {Bot }}}$, can be calculated (ref. Equation (31)), and that of $\mathrm{SE}^{\text {Bot }}$ in Figure 9d can be obtained from the assembly of $\mathbf{K}_{\mathrm{SE}_{1}^{\text {Bot }}}$ and $\mathbf{K}_{\mathrm{SE}_{2}^{\text {Bot }}}$ (ref. Equation (32)).

If both the top and bottom matrix cracks are present, then both $\mathrm{SE}^{\mathrm{Top}}$ and $\mathrm{SE}^{\mathrm{Bot}}$ are integrated. Their integrations are performed over the same domain, i.e., the domain of the whole cohesive element. In this case, it is assumed that the solution is a superposition of those of the two SEs, each carrying half of the weight. The final stiffness matrix of the whole cohesive element is therefore the weighted superposition of 

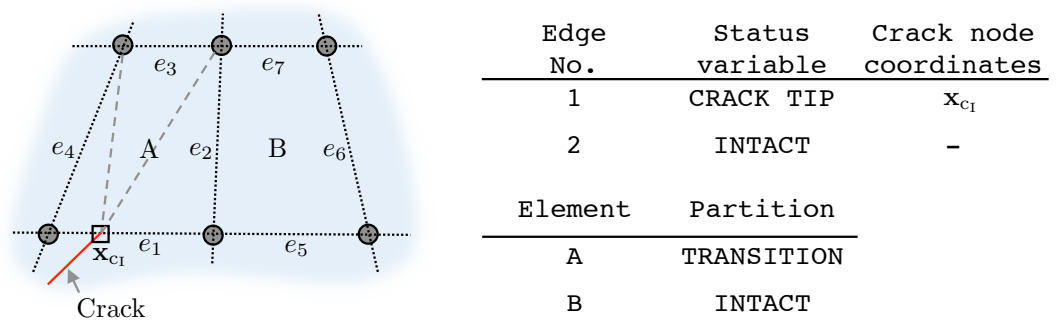

(a) Before crack propagation across Element A.

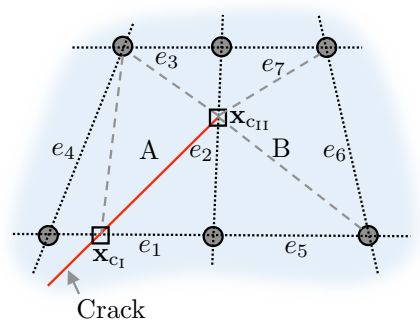

\begin{tabular}{ccc}
$\begin{array}{c}\text { Edge } \\
\text { No. }\end{array}$ & $\begin{array}{c}\text { Status } \\
\text { variable }\end{array}$ & $\begin{array}{c}\text { Crack node } \\
\text { coordinates }\end{array}$ \\
\hline 1 & CRACK WAKE & $\mathbf{x}_{\mathrm{C}_{I}}$ \\
2 & CRACK TIP & $\mathbf{x}_{\mathrm{C}_{I I}}$ \\
Element & Partition & \\
A & CRACKED & \\
B & TRANSITION
\end{tabular}

(b) After crack propagation across Element A.

Figure 10: An edge status variable approach for modelling crack propagation in a mesh.

those of $\mathrm{SE}^{\mathrm{Top}}$ and $\mathrm{SE}^{\mathrm{Bot}}$ :

$$
\mathbf{K}_{\mathrm{CE}}=\mathcal{A}\left(\frac{1}{2} \mathbf{K}_{\mathrm{SE}^{\mathrm{Top}}}, \quad \frac{1}{2} \mathbf{K}_{\mathrm{SE}}{ }^{\text {Bot }}\right)
$$

\subsection{Crack propagation: edge status variable approach}

Matrix cracks are here modelled with the cohesive zone model. The cohesive zone lies within several cohesive elements, and its exact boundaries are not explicitly represented in the mesh. The "numerical crack tip" (here defined by the forefront of the cohesive elements along the direction of propagation) is only required to be ahead of the cohesive zone and its exact location does not need to be precisely determined. For simplicity, the numerical crack tip is propagated from one edge to another in the mesh. With the edge connectivity available for each element, an edge status variable approach is developed for the modelling of cohesive crack propagations. A list of all the edges is created, where a status variable, $\mu$, and a coordinates vector, $\mathbf{x}_{\mathrm{c}}$, are allocated to every edge. $\mu$ stores the current status of the edge, i.e., intact, hosting a crack tip, or already at the wake of the crack tip. $\mathbf{x}_{\mathrm{c}}$ stores the coordinates of the crack node on the edge. With the edge status variable approach, elements can read and update the variables of their own edges and partitions into SEs accordingly, and the cohesive cracks are naturally propagated across neighbouring elements. The example in Figure 10 demonstrates how a matrix crack is propagated across two ply elements (shown in plan view). The numerical crack tip initially resides on Edge 1 at the location $\mathbf{x}_{\mathrm{c}_{\mathrm{I}}}$, and Element $\mathrm{A}$ is in a transition-element partition, where three wedge SEs are formed around the crack tip (Figure 10a). After a certain propagation criterion is satisfied, the status variable of Edge 1 is updated from "CRACK TIP" (i.e., hosting a crack tip) to "CRACK WAKE" (i.e., at the wake of the crack). Element A then calculates the new crack tip coordinates, $\mathbf{x}_{\mathrm{C}_{\mathrm{II}}}$, and updates its partition from a transition element to a cracked element, where four wedge SEs and one cohesive SE are formed to represent the cohesive crack within the element (Figure 10b). It also updates the status variable of Edge 2 from "INTACT" to "CRACK TIP", and stores 


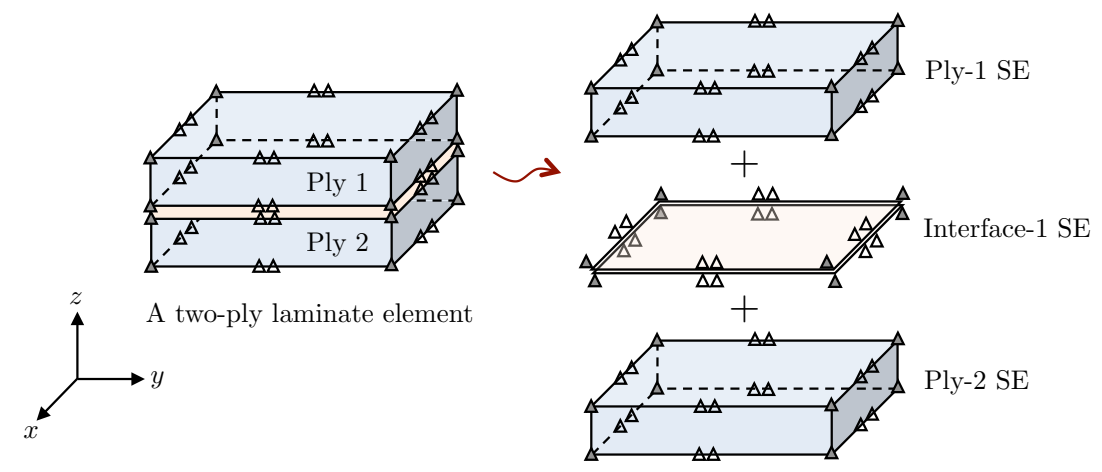

Figure 11: A laminate element can be constructed based on layup

$\mathbf{x}_{\mathrm{C}_{\text {II }}}$ as the crack node coordinates on this edge (Figure 10b). After reading the edge status and crack node coordinate variables of Edge 2, Element B knows that a crack tip has arrived on Edge 2. It can then partition itself into a transition element, where three SEs are formed around the crack tip on Edge 2 (Figure 10b). In this way, an element only needs to read a fixed amount of information to propagate a crack, i.e., the edge status variables of its own edges, regardless of the total number of cracks in the mesh. Every element can propagate a crack in this domain, and a large number of cracks can be modelled in the mesh. This capability will be demonstrated in the applications afterwards.

\subsection{A laminate element}

With the enriched ply and cohesive elements defined in the previous sections, a laminate element can be formed, such that the ply and cohesive elements are SEs of the laminate element (Figure 11). Hence, multiple plies and interfaces are represented within such a laminate element, together with the matrix cracks, delamination and their intersections, represented respectively by the ply SEs and interface cohesive SEs as described in the previous sections. The construction of a FNM laminate element is presented in this section. From the layup and the single ply thickness, the nodal coordinates of the ply and interface SEs can be easily calculated, the procedure of which is omitted here for brevity. The allocation of DoF sets is also straightforward. Let $N_{\text {dofPLY }}$ represent the number of DoF sets (both nodal and floating) of an enriched ply element, and $N_{\text {dofINTERF }}$ represent that of an enriched cohesive element for interface. A laminate element for $n$ ply-blocks would have the number of DoF sets, $N_{\text {dofLAM }}$, to be:

$$
N_{\text {dofLAM }}=n \times N_{\text {dofPLY }}
$$

The $i$ th ply SE would simply have the $i$ th group of $N_{\text {dofPly }}$ DoF sets in its DoF array, DofArray ${ }_{\mathrm{PLY}} i$ :

$$
\operatorname{DofArray}_{\mathrm{PLY} i}(j)=(i-1) \times N_{\mathrm{dofPLY}}+j, \text { where } j=1,2, \ldots, N_{\mathrm{dofPLY}}
$$

The $i$ th interface cohesive SE shares the top surface DoF sets of the $i$ th ply element and the bottom surface DoF sets of the $(i+1)$ th ply element. Therefore, it would have the following DoF sets in its DoF array, 


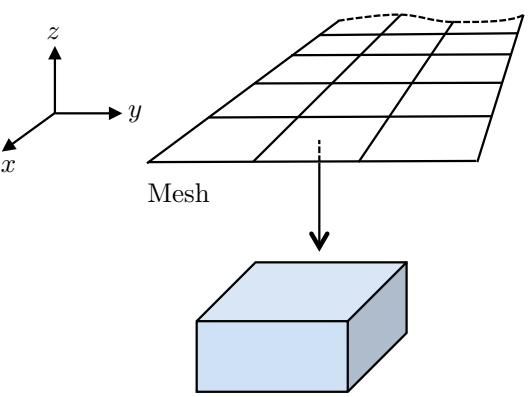

A laminate element

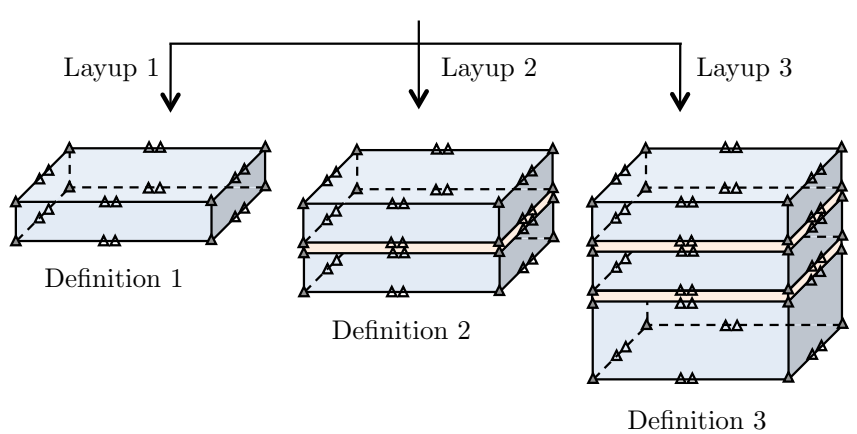

Figure 12: A common planar mesh can be used for arbitrary layups. A different laminate element is defined for each different layup.

DofArray $_{\text {INTERF } i}$ :

$$
\begin{array}{cl}
\operatorname{DofArray}_{\operatorname{INTERF} i}(l) & =\operatorname{DofArray}_{\mathrm{PLY} i}\left(l+N_{\mathrm{dofPLY}} / 2\right), \\
\operatorname{DofArray}_{\operatorname{INTERF} i}\left(l+N_{\text {dofINTERF }} / 2\right) & =\operatorname{DofArray}_{\mathrm{PLY} i+1}(l),
\end{array}
$$

where $l=1 \sim N_{\text {dofInTERF }} / 2$. With the above-defined connectivities, all the DoF sets in the laminate element can be allocated to the respective ply and interface cohesive SEs for their calculations as detailed in the previous sections. Their system equations are then assembled to form that of the laminate element. Note that all the SEs are within the same laminate element. The information of one SE is directly available to another. For example, the stress, strain, energy, failure status variables, etc., of the ply SEs are directly available to the interface SEs, and the latter can then be programmed to apply corresponding failure criteria or form corresponding partitions to explicitly represent the matrix crack boundary on the interface [46] or to migrate the delamination towards a certain ply [49]. In this work, the edge status variables and crack node coordinates of ply SEs will be passed into the interface SEs, such that the latter can be partitioned to explicitly represent the matrix crack boundaries, as detailed in Section 3.2. The use of such a laminate element greatly reduces the effort of preprocessing. The layup and ply thickness are defining parameters of this element, and they do not need to be reflected in the mesh. Therefore, a single-layer, planar mesh can be used for arbitrary layups. A different laminate element is formed for each different layup (Figure 12).

\subsection{Some implementation details}

In the current implementation of the FNM, a crack is assumed to always cross an edge of an element. If the crack path comes very close to a node, it is deviated such that the crack node lies on the edge (see 


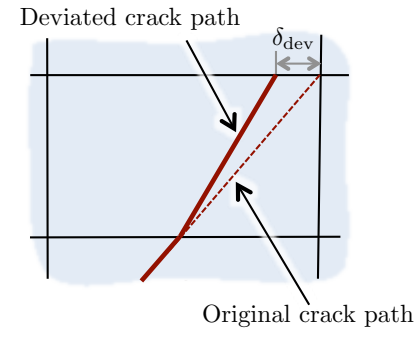

(a) Deviation of crack path around a node.

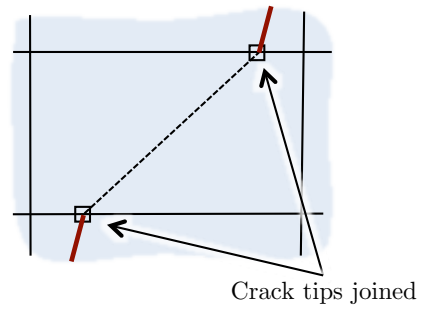

(b) Joining of two crack nodes in one element.

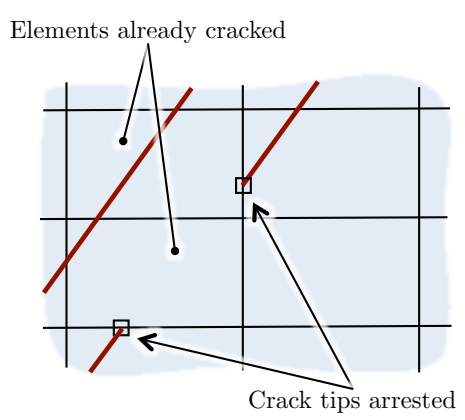

(c) Arrest of crack tips on edges of cracked elements.

Figure 13: Some additional details

Figure 13a). The length of deviation, $\delta_{\mathrm{dev}}$, is set to be $10 \%$ of the length of the eventually cracked edge. Note that similar approaches have been used in XFEM [48].

Matrix cracks may initiate from different locations (e.g., two different edges) and, after a certain duration of propagation, reach the boundaries of the same element. In this case, it is assumed that the two cracks join up in this element, and the element is partitioned such that the two crack tips are connected (see Figure 13b). This would inevitably compromise the direction of the crack in the element. However, the number of such elements is small compared to the total number of elements in the mesh, and the effect of the local deviation of crack direction in these elements on the overall prediction of failure is deemed limited.

In the FNM, every element is capable of modelling a crack within its domain. No empirical limit is set for the maximum number of matrix cracks modelled in the mesh. Together with the edge status variable approach, a high density of matrix cracks can be modelled. However, when the mesh is too coarse, the stress relaxation between two cracks are not properly captured. It may occur that after a crack has crossed an element and the element has been partitioned, a neighbouring crack may again reach the boundary of the element. In this case, for simplicity, the second crack is arrested (i.e., crack tip node is tied to the intact edge) on the boundary and no further propagation is considered (see Figure 13c). Otherwise, a refined mesh would have intact elements between two neighbouring cracks, where the strain energy is insufficient to propagate another crack in-between. In principle, the FNM can allow for multiple parallel cracks to form inside an element. It is a matter of including sufficient DoF and coding the corresponding partitioning for integration. Since increasing the maximum allowed crack density can also be achieved via mesh refinement of the FE model, the partitioning for multiple parallel cracks inside an element has not been implemented.

\subsection{Towards a generic $3 D$ element definition}

There is no theoretical barrier in extending the FNM to the modelling of more complicated cracking scenarios. In addition to the node and edge connectivities, a generic 3D element could also have a surface connectivity as part of the element definition (Figure 14a). Floating DoF sets could then be assigned to the edges, surfaces, and volume of an element, as shown in Figure 14b. By employing the correct sets of floating DoFs and forming the SEs with respect to the cracks, the element in Figure 14b could model complex intersecting cracks (Figure 15). In addition, the SEs may use other methods of interpolation and integration, e.g., the Smoothed Finite Element Method (SFEM) [51]. 

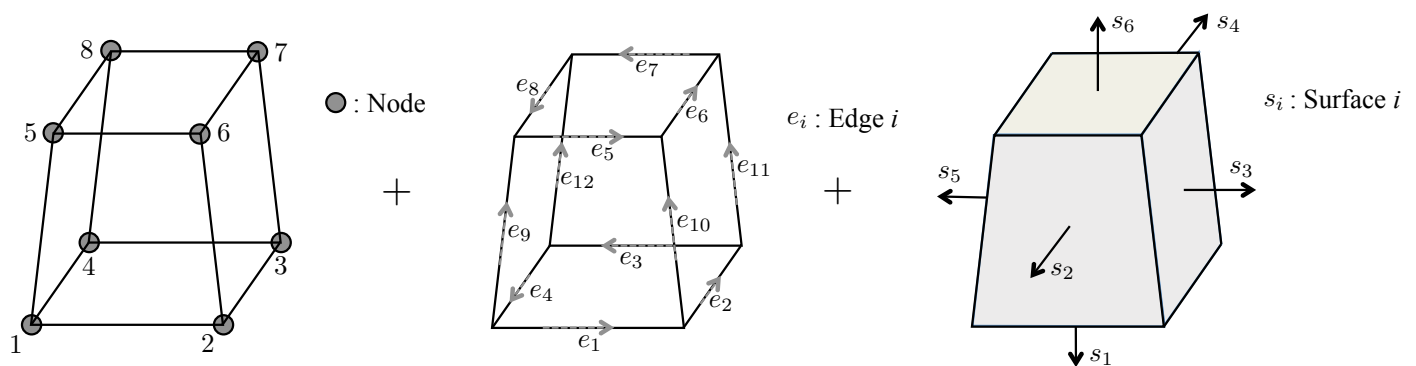

(a) The definition of a 3D element in FNM includes node, edge and surface connectivities.

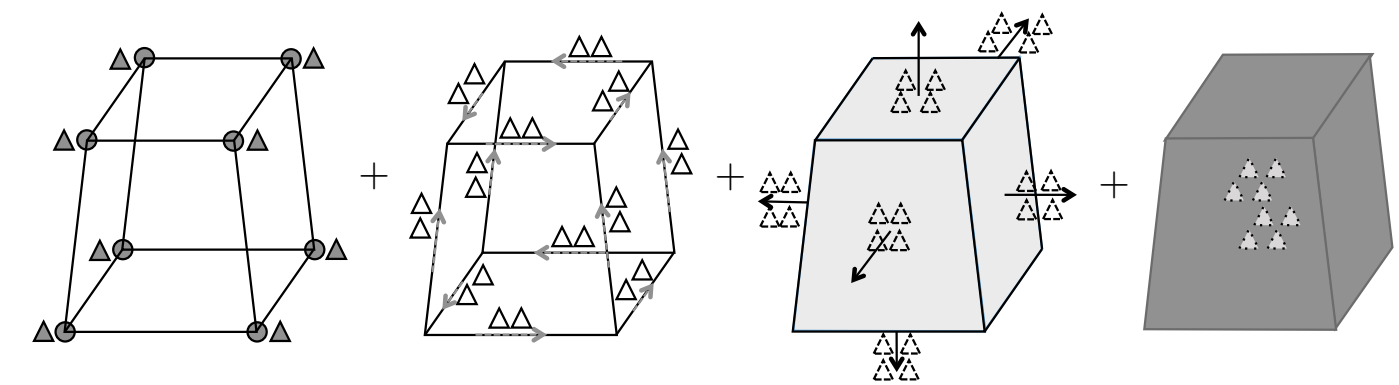

O: Node

$\triangle:$ Nodal DoF

$\triangle$ : Edge Floating DoF

公: Surface Floating DoF

$\therefore$ : Volume (internal) Floating DoF

(b) The DoF allocations of a 3D element in FNM.

Figure 14: Generalization of FNM to a 3D element

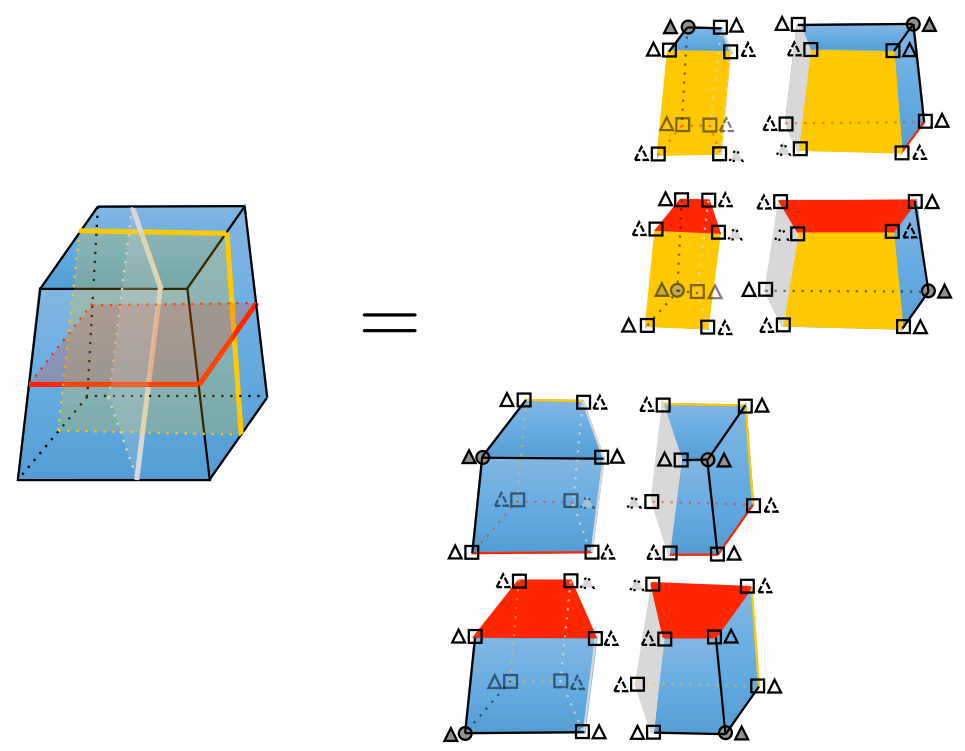

Figure 15: Modelling three intersecting cracks with edge, surface and volume floating DoF sets 


\section{Failure theories}

Different failure theories can be used on the elements defined in the previous section. In this work, a maximum stress criterion is used to predict the onset of fibre tensile failure:

$$
\frac{\sigma_{1}}{X_{\mathrm{t}}}=1,
$$

where $X_{\mathrm{t}}$ is the fibre-directional tensile strength. Fibre compressive failure is not considered in this work. The standard, bilinear cohesive law is used to model the damage evolution of fibre failure:

$$
\int_{0}^{u_{1}^{\mathrm{f}}} \sigma_{1} \mathrm{~d} u_{1}=\frac{1}{2} X_{\mathrm{t}} u_{1}^{\mathrm{f}}=G_{\mathrm{fc}},
$$

where $u_{1}^{\mathrm{f}}$ is the fibre-directional displacement $\left(u_{1}\right)$ at total failure. Note that the translaminar fracture toughness, $G_{\mathrm{fc}}$, scales with the thickness of the ply-block (ref. Equation (21)). A damage variable, $d_{\mathrm{f}}, 0 \leq$ $d_{\mathrm{f}} \leq 1$, can be defined from the cohesive law curve to characterize the softening due to fibre damage:

$$
\begin{aligned}
E_{1}^{\prime} & =E_{1} \times\left(1-d_{\mathrm{f}}\right), \\
\nu_{12}^{\prime} & =\nu_{12}\left(1-d_{\mathrm{f}}\right), \\
\nu_{13}^{\prime} & =\nu_{13}\left(1-d_{\mathrm{f}}\right),
\end{aligned}
$$

where the non-primed moduli are the original ones and the primed the softened ones.

If the fibre failure onset criterion in Equation (37) is met, then it is assumed that the breaking of fibres would also cause fibre-matrix debonding and matrix cracking. Therefore, the fibre failure onset criterion also constitutes a criterion for matrix failure onset. In the absence of fibre failure onset, the matrix tensile failure onset is determined by the quadratic interactive criterion based on the normal and shear tractions on the fracture surface:

$$
\left(\frac{\tau_{\mathrm{n}}}{\tau_{\mathrm{n}}^{\mathrm{c}}}\right)^{2}+\left(\frac{\tau_{\mathrm{t}}}{\tau_{\mathrm{t}}^{\mathrm{c}}}\right)^{2}+\left(\frac{\tau_{\ell}}{\tau_{\ell}^{\mathrm{c}}}\right)^{2}=1
$$

where $\tau_{\mathrm{n}}, \tau_{\mathrm{t}}$ and $\tau_{\ell}$ are the normal and two (longitudinal and transverse) shear tractions on the matrix crack surface, respectively; and $\tau_{\mathrm{n}}^{\mathrm{c}}, \tau_{\mathrm{t}}^{\mathrm{c}}$ and $\tau_{\ell}^{\mathrm{c}}$ are the corresponding strengths. Matrix compressive failure is not considered in this work. It is assumed that matrix cracks are all perpendicular to the lamina plane in tensile failure. Therefore, $\tau_{\mathrm{n}}=\sigma_{2}, \tau_{\mathrm{t}}=\tau_{13}$, and $\tau_{\ell}=\tau_{12}$. Once the matrix failure onset occurs (determined either by Equation (37) or by Equation (40)), the element is partitioned such that a cohesive SE models the cohesive crack in the element domain, as detailed in Section 3.1. The failure onset of this cohesive SE is determined by the same criterion as above. The damage evolution of the cohesive SE follows a mixed-mode (power-law) bilinear cohesive law:

$$
\int_{0}^{\delta_{\text {eff }}^{\mathrm{f}}} \tau_{\text {eff }} \mathrm{d} \delta_{\mathrm{eff}}=\frac{1}{2} \tau_{\text {eff }}^{\max } \delta_{\text {eff }}^{\mathrm{f}}=G_{\mathrm{mc}}
$$

where:

$$
\delta_{\text {eff }}=\sqrt{\left\langle\delta_{\mathrm{n}}\right\rangle^{2}+\delta_{\mathrm{t}}^{2}+\delta_{\ell}^{2}}, \quad \tau_{\mathrm{eff}}=\frac{\tau_{\mathrm{n}}\left\langle\delta_{\mathrm{n}}\right\rangle+\tau_{\mathrm{t}} \delta_{\mathrm{t}}+\tau_{\ell} \delta_{\ell}}{\delta_{\mathrm{eff}}}, \quad\left\langle\delta_{\mathrm{n}}\right\rangle=\max \left(0, \delta_{\mathrm{n}}\right) .
$$


$\delta_{\mathrm{n}}, \delta_{\mathrm{t}}$, and $\delta_{\ell}$ are the normal, transverse and longitudinal separations of the cohesive crack surfaces, respectively. $\tau_{\text {eff }}^{\max }$ is the maximum value of $\tau_{\text {eff }}$, i.e., the value of $\tau_{\text {eff }}$ at failure onset. The tractions relate to the separations through the following equation:

$$
\left\{\begin{array}{c}
\tau_{\mathrm{n}} \\
\tau_{\ell} \\
\tau_{\mathrm{t}}
\end{array}\right\}=\left[\begin{array}{ccc}
K_{\mathrm{n}}\left(1-d_{\mathrm{m}}\right) & 0 & 0 \\
0 & K_{\ell}\left(1-d_{\mathrm{m}}\right) & 0 \\
0 & 0 & K_{\mathrm{t}}\left(1-d_{\mathrm{m}}\right)
\end{array}\right]\left\{\begin{array}{l}
\delta_{\mathrm{n}} \\
\delta_{\ell} \\
\delta_{\mathrm{t}}
\end{array}\right\},
$$

where $K_{\mathrm{n}}, K_{\ell}$ and $K_{\mathrm{t}}$ are the penalty stiffness and $d_{\mathrm{m}}$ is the damage variable defined based on the cohesive law curve. $G_{\mathrm{mc}}$ is the power-law mixed-mode fracture toughness defined at failure onset as:

$$
G_{\mathrm{mc}}=\left[\left(\frac{\lambda_{\mathrm{n}}}{G_{\mathrm{nc}}}\right)^{\alpha}+\left(\frac{\lambda_{\mathrm{t}}}{G_{\mathrm{tc}}}\right)^{\alpha}+\left(\frac{\lambda_{\ell}}{G_{\ell \mathrm{c}}}\right)^{\alpha}\right]^{-1 / \alpha}
$$

where $\alpha$ is the power law exponent, a material property to be determined experimentally; $G_{\mathrm{nc}}, G_{\mathrm{tc}}$ and $G_{\ell \mathrm{c}}$ are the fracture toughness in normal, transverse shear and longitudinal shear directions, respectively. $\lambda_{\mathrm{n}}$, $\lambda_{\mathrm{t}}$ and $\lambda_{\ell}$ are determined at failure onset to be:

$$
\lambda_{\mathrm{n}}=\frac{G_{\mathrm{n}}}{G_{\mathrm{m}}}, \quad \lambda_{\mathrm{t}}=\frac{G_{\mathrm{t}}}{G_{\mathrm{m}}}, \quad \lambda_{\ell}=\frac{G_{\ell}}{G_{\mathrm{m}}}, \quad G_{\mathrm{m}}=G_{\mathrm{n}}+G_{\mathrm{t}}+G_{\ell},
$$

where:

$$
G_{\mathrm{n}}=\tau_{\mathrm{n}}\left\langle\delta_{\mathrm{n}}\right\rangle, \quad G_{\mathrm{t}}=\tau_{\mathrm{t}} \delta_{\mathrm{t}}, \quad G_{\ell}=\tau_{\ell} \delta_{\ell} .
$$

The propagation criterion of matrix crack (Section 3.3) is based on both the failure onset status of the cohesive SE at the wake of the numerical crack tip, and those of the SEs of the transition element ahead of the crack tip (see Figure 10). If any of the SEs reaches failure onset, the progagation criterion is met, and the cohesive crack is propagated using the edge status variable approach, detailed in Section 3.3.

Similarly to matrix cracking, delamination onset in the cohesive elements and SEs is determined by the same quadratic interactive criterion in Equation (40). The damage evolution in delamination also follows the mixed-mode (power-law) bilinear cohesive law detailed in Equation $(41) \sim(46)$.

\section{Applications}

The elements and the failure theories presented in the previous sections are applied on the modelling of the two problems introduced in Section 1, Figure 1. They are on the tensile failure of notched and unnotched $\left[45_{4} / 90_{4} /-45_{4} / 0_{4}\right]_{\mathrm{S}} \mathrm{IM} 7 / 8552$ carbon/epoxy laminates, respectively $[1,2]$. The material properties used for the simulations are summarized in Table 1. The enriched elements in Section 3 are implemented as user-defined elements in the commercial finite element package Abaqus. All analysis in this section are quasi-static, performed using the implicit method.

\subsection{Tensile failure of notched $\left[45_{4} / 90_{4} /-45_{4} / 0_{4}\right]_{\mathrm{s}}$ laminate}

The progressive failure of the Open-Hole Tension (OHT) of $\left[45_{4} / 90_{4} /-45_{4} / 0_{4}\right]_{\mathrm{s}}$ laminate in [2] is modelled in this section. The experimental image in Figure 1a shows that the laminate has disintegrated at final failure, where the delamination has completely separated the angle plies with the 0 plies. The 
Table 1: Material properties of IM7/8552 carbon/epoxy composite [22].

\begin{tabular}{ll}
\hline Property & Value \\
\hline Longitudinal Young's modulus: $E_{1}(\mathrm{GPa})$ & 161 \\
Transverse Young's modulus: $E_{2}, E_{3}(\mathrm{GPa})$ & 11.4 \\
Shear modulus: $G_{12}, G_{13}(\mathrm{GPa})$ & 5.17 \\
Shear modulus: $G_{23}(\mathrm{GPa})$ & 3.98 \\
Poisson's ratio: $\nu_{12}, \nu_{13}$ & 0.32 \\
Poisson's ratio: $\nu_{23}$ & 0.43 \\
Longitudinal tensile strength: $X_{\mathrm{t}}(\mathrm{MPa})$ & 2806 \\
Transverse tensile strength: $\tau_{\mathrm{n}}^{\mathrm{c}}(\mathrm{MPa})$ & 60 \\
Shear strength: $\tau_{\mathrm{t}}^{\mathrm{c}}, \tau_{\ell}^{\mathrm{c}}(\mathrm{MPa})$ & 90 \\
Interfacial normal strength: $\tau_{\mathrm{n}}^{\mathrm{c}}(\mathrm{MPa})$ & 40 \\
Interfacial shear strength: $\tau_{\mathrm{t}}^{\mathrm{c}}, \tau_{\ell}^{\mathrm{c}}(\mathrm{MPa})$ & 50 \\
Single-ply translaminar fracture toughness: $G_{\mathrm{fc}}^{1}\left(\mathrm{~kJ} / \mathrm{m}^{2}\right)$ & 112.7 \\
Mode I matrix/interfacial fracture toughness: $G_{\mathrm{nc}}\left(\mathrm{kJ} / \mathrm{m}^{2}\right)$ & 0.293 \\
Mode II matrix/interfacial fracture toughness: $G_{\mathrm{tc}}, G_{\ell \mathrm{c}}\left(\mathrm{kJ} / \mathrm{m}^{2}\right)$ & 0.631 \\
Power-law exponent for mixed-mode fracture: $\alpha$ & $1($ assumed $)$ \\
Penalty stiffness of cohesive elements or SEs: $K_{\mathrm{n}}, K_{\ell}, K_{\mathrm{t}}\left(\mathrm{N} / \mathrm{mm}^{3}\right)$ & $10^{6}[11]$ \\
\hline
\end{tabular}

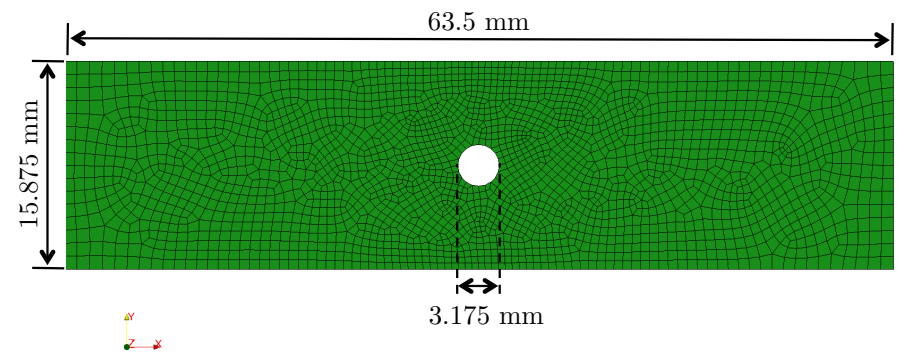

(a) Fnite element mesh for the OHT quasi-isotropic laminate

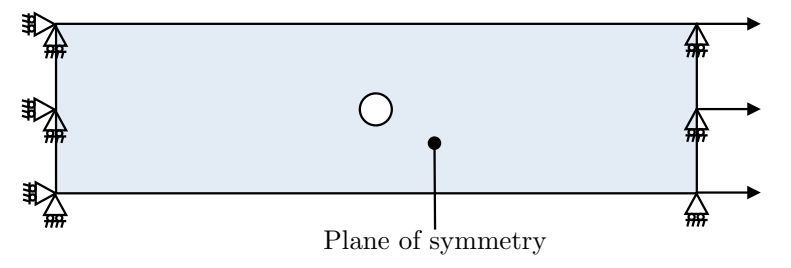

(b) Boundary conditions for the OHT quasi-isotropic laminate

Figure 16: Finite element model of OHT laminate under tension. 
experimental observations in [2] report that the failure of the 0 plies is similar to that of two unidirectional plates, where the explosive development of longitudinal splits occur together with the breaking of fibres (Figure 2b).

The finite element mesh is shown in Figure 16a. Note that a planar mesh is used, no meshing in the thickness direction is needed. A randomly-generated, unstructured mesh is purposely used to demonstrate the effectiveness of the FNM on arbitrary meshes. Hence, minimal preprocessing is required to build the model. Each element is a laminate element with 4 ply-blocks and 3 interfaces, defined based on the layup (ref. Section 3.4). The elements are about $0.5 \mathrm{~mm} \times 0.5 \mathrm{~mm}$ in dimension in the region around the hole, and $1 \mathrm{~mm} \times 1 \mathrm{~mm}$ in the regions away from the hole. The boundary conditions of this problem are shown in Figure 16b. Note that only half of the laminate, i.e., $\left[45_{4} / 90_{4} /-45_{4} / 0_{4}\right]$, is considered, with the symmetric boundary condition applied on the laminate midplane. The transverse displacement is constrained at the grip ends to approximate the effect of gripping. The predicted strength of the laminate is $295 \mathrm{MPa}$, which agrees well with the experimental average of $275 \mathrm{MPa}$ [2]. The simulated damage patterns are summarized in Figure 17. The red area shows cohesive elements/SEs which have reached total failure, i.e., $d_{\mathrm{m}}=1$ (ref. Equation (43)).

Matrix cracks and splits are predicted to appear firstly around the hole, followed by delamination mainly on the 45/90 and 90/-45 interfaces (Figure 17a). The delamination area coincides with the locations of matrix cracks, indicating that the enriched cohesive elements on the interface successfully captured the local delamination induced by the matrix cracks. As the loading continues, $-45^{\circ}$ cracks develop across the width of the laminate, and the $0^{\circ}$ splits continue to propagate towards the grips. These cracks drive the delamination to propagate on the $-45 / 0$ interface (Figure 17b). This delamination covers a significant area of the laminate, which causes the load-drop. A "staircase" delamination pattern can be observed as shown in Figure 18, where delamination follows the boundaries set by the matrix cracks. In Figure 19, the experimental X-Ray image of all the damages (superimposed) is compared with the predicted damage patterns, and the X-Ray images of delamination on individual interfaces are also compared with the predicted delamination patterns. A close agreement is reached between the experimental images and the predicted patterns. Further loading causes the initial $0^{\circ}$ splits to propagate all the way until the grips, together with the massive occurrence of splits in other locations which separate the 0 ply-block into loosened strips (Figure $17 \mathrm{c}$ ). The $45^{\circ}, 90^{\circ}$ and $-45^{\circ}$ matrix cracks are widely opened. While the delamination on the 45/90 and 90/-45 interfaces remains stagnant, that on the $-45 / 0$ interface propagates across almost the entire length of the laminate, causing the complete separation of the angle plies with the 0 plies. Continued loading would increase the separation of the angle plies with the 0 plies, evidenced by the out-of-plane displacement contour in Figure 20a. Eventually, fibre failures in the 0 plies cause the transverse bulging of the broken strips (Figure 20b). Note that the predicted fibre failures are rather distributed, which signifies that they are the results of the individual breaking of the loosened strips. A close examination of the post-failure image in Figure 1a suffices to tell that this is indeed the case in the experiment. The authors believe that this complete sequence of progressive failure events, up to the final breaking of the loosened $0^{\circ}$ strips, is for the first time captured by simulations.

\subsection{Tensile failure of unnotched $\left[45_{4} / 90_{4} /-45_{4} / 0_{4}\right]_{\mathrm{s}}$ laminate}

The progressive tensile failure of the unnotched $\left[45_{4} / 90_{4} /-45_{4} / 0_{4}\right]_{s}$ laminate in [1] is simulated in this section. Similarly to the previous case, the experimental image in Figure 1b shows that the laminate has disintegrated at final failure, where the delamination has completely separated the angle plies with 


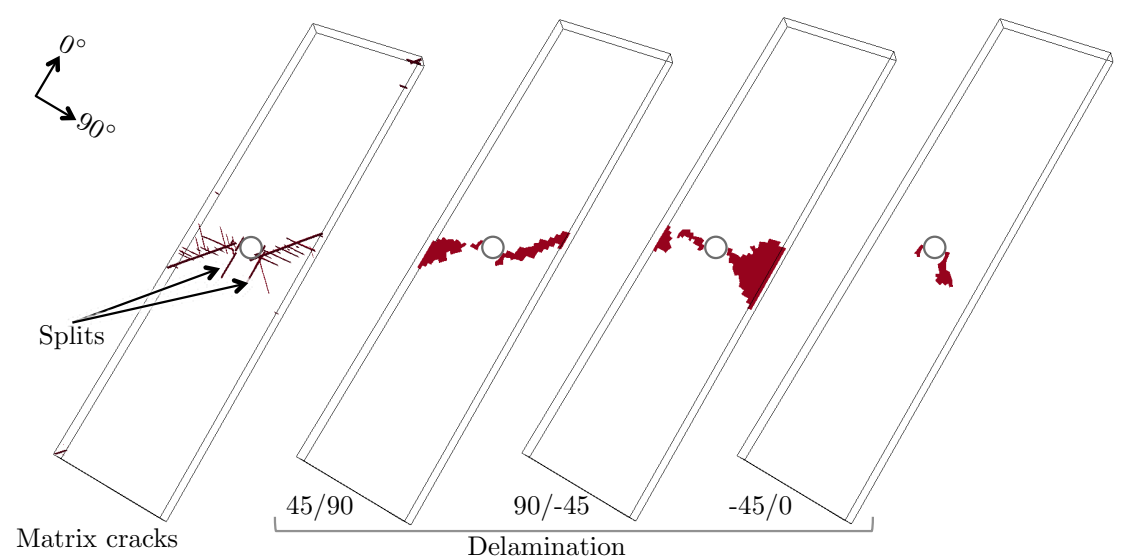

(a) Initial-stage failure patterns.

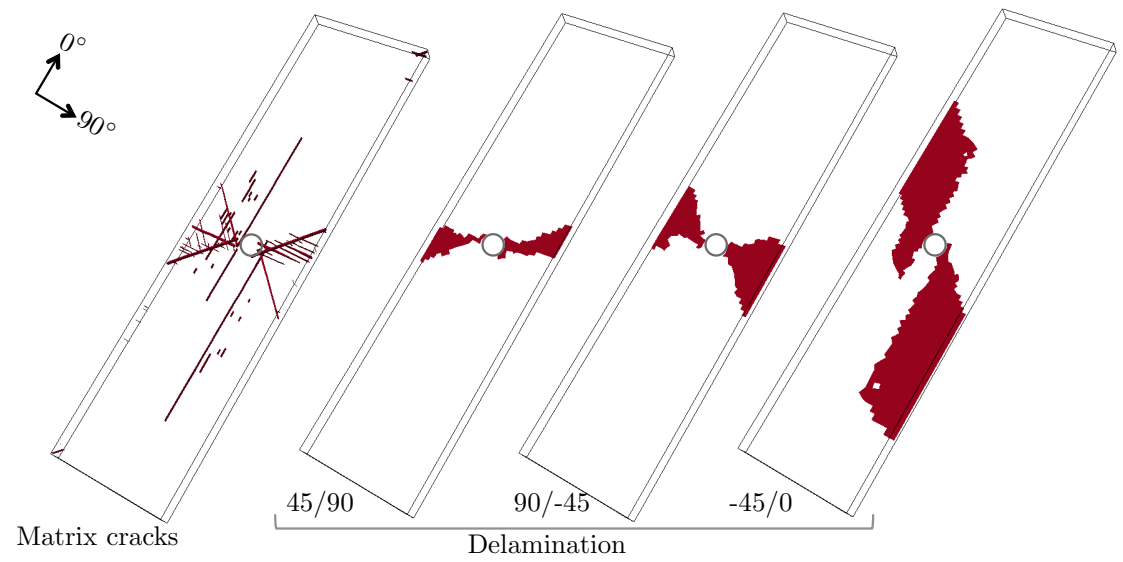

(b) Mid-stage failure patterns.

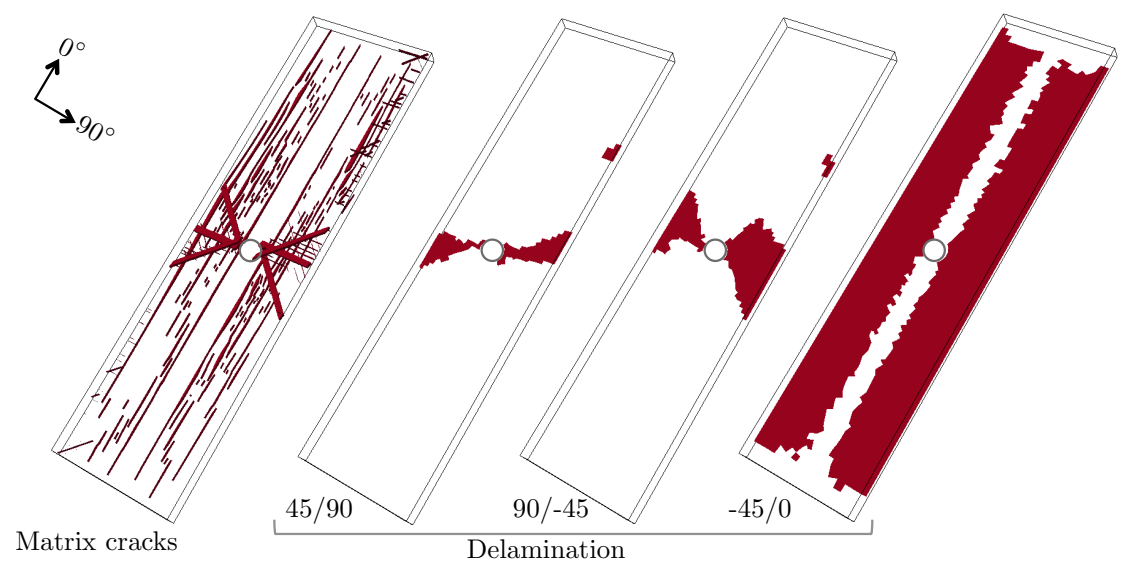

(c) Final failure patterns.

Figure 17: Failure pattern predictions of the OHT $\left[45_{4} / 90_{4} /-45_{4} / 0_{4}\right]_{\mathrm{s}}$ laminate in [2]. 


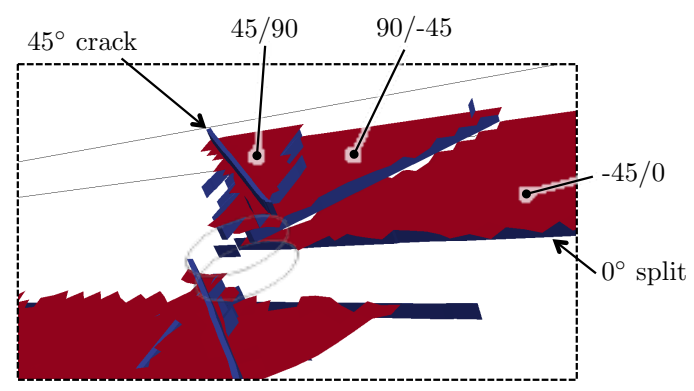

Figure 18: The "staircase" delamination patterns.

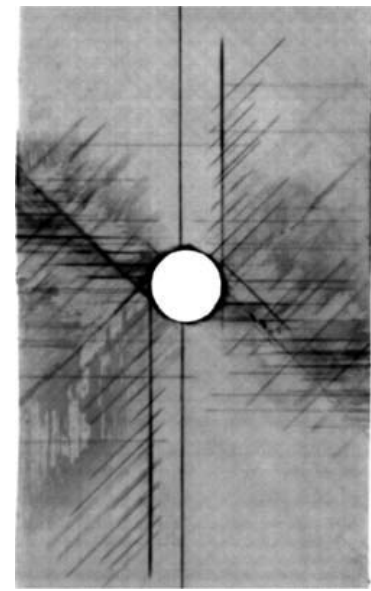

(a) Experimental X-Ray image of damages in the laminate [1].

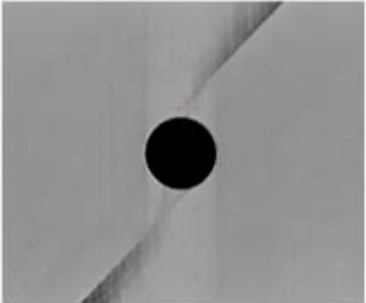

$45 / 90$

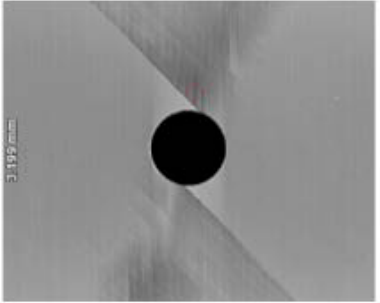

$90 /-45$

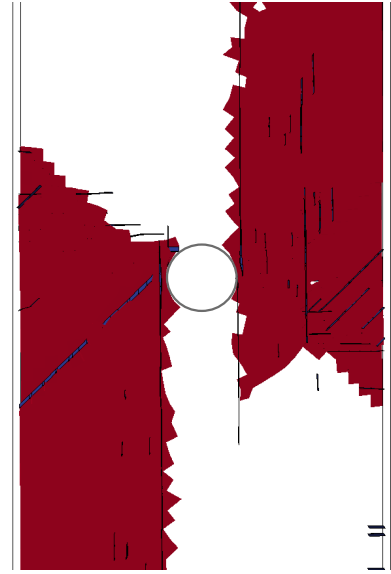

(b) Simulated damage patterns.

(c) Experimental X-Ray image of delamination on the three interfaces [26].

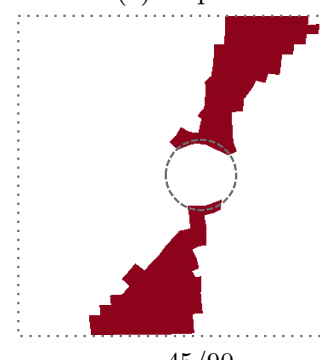

$45 / 90$

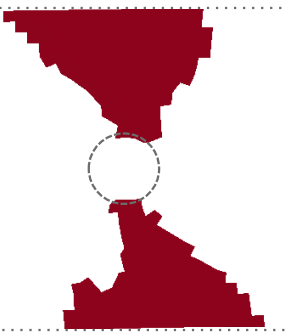

$90 /-45$

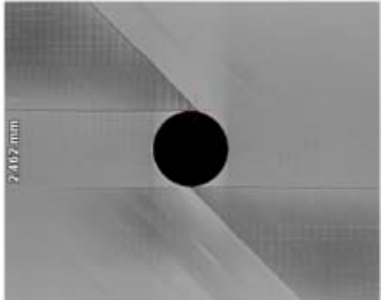

$-45 / 0$

(d) Simulated delamination patterns on the three interfaces.

Figure 19: X-Ray damage images vs predicted damage patterns 


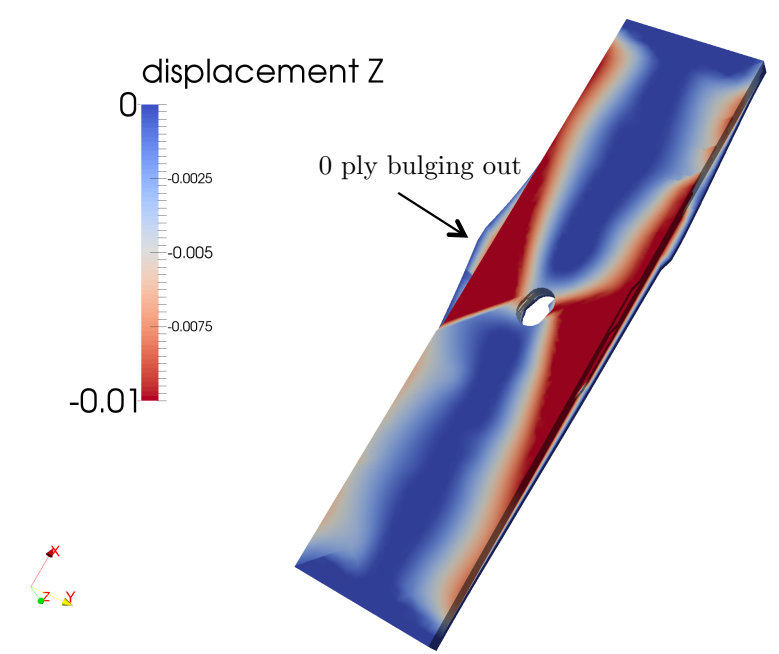

(a) The predicted out-of-plane displacement contour shows the disintegration of the laminate, and the transverse bulging of the failed 0 plies.

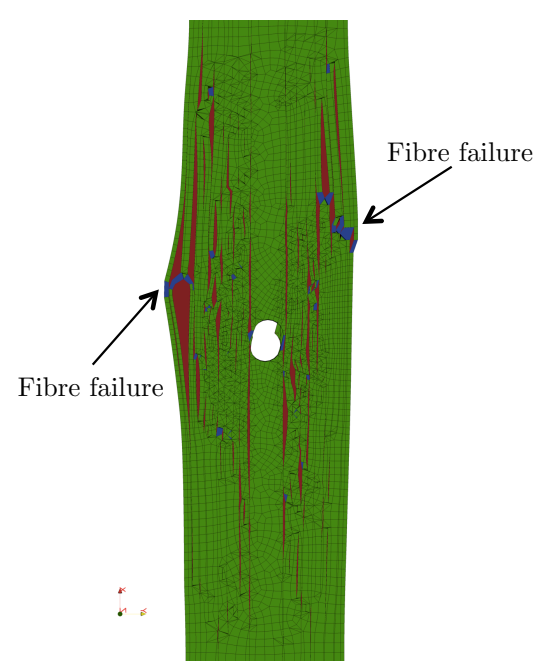

(b) Fibre failure (blue-colored) in the 0 plies causes the transverse bulging of the broken pieces.

Figure 20: Final failure displacement contour and fibre failure in the 0 plies

the 0 plies. The experimental observations in [1] report that the failure of 0 plies initiated from the grip, accompanied by the development of extensive longitudinal splits.

The finite element mesh is shown in Figure 21a. Similarly to the previous case, a planar mesh is used, which is made of the laminate elements with 4 ply-blocks and 3 interfaces, defined based on the layup (ref. Section 3.4). All the elements are about $1 \mathrm{~mm} \times 1 \mathrm{~mm}$ in dimension. The boundary conditions of this problem are shown in Figure 21b. The predicted strength of the laminate is $526 \mathrm{MPa}$, which agrees well with the experimental average of $541 \mathrm{MPa}$ [1]. The simulated damage patterns are summarized in Figure 22. The red area shows cohesive elements/SEs which have reached total failure, i.e., $d_{\mathrm{m}}=1$ (ref. Equation (43)).

Matrix cracks are predicted to appear firstly in the surface 45 plies, followed by the occurrence of $90^{\circ}$ matrix cracks along the $45^{\circ}$ crack paths and also on the edges. $45 / 90$ delamination is mainly predicted at locations of the $45^{\circ}$ matrix cracks. 90/-45 delamination also appears at certain locations along the edges, in approximately triangular shapes. This agrees well with the experimental record in [1]. Figure 22a shows the predicted initial matrix cracks and delamination patterns.

As the loading continues, matrix cracks continue to grow in quantity in all the angle plies. Three zones with a high concentration of matrix cracks can be identified in the predicted matrix crack patterns in Figure 22b. Correspondingly, the predicted patterns of 45/90 delamination in Figure 22b follow closely those of the three zones of matrix cracks. Significant delamination is predicted to occur on the 90/-45 interface. Such a phenomenon is also observed in the experiment in [1]. The out-of-plane displacement contour prediction in Figure 23 clearly shows the peel-off of both the 45 and the 90 plies. At this stage, it appears that all three zones have similar densities of matrix cracking, with Zone B seemingly having a slightly larger amount of matrix cracks. In terms of delamination, Zone $\mathrm{C}$ seems to have a relatively smaller amount of overall delamination compared to Zone A and Zone B, particularly on the $-45 / 0$ interface.

The final failure of the laminate is marked with the sudden appearance of fibre failure in the grip region in Zone $\mathrm{C}$ and a longitudinal split which spans across more than $2 / 3$ of the length of the laminate. The 


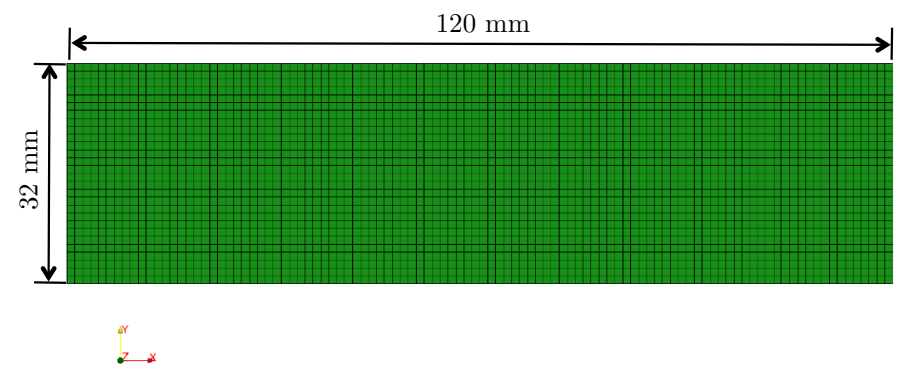

(a) Fnite element mesh for the unnotched quasi-isotropic laminate

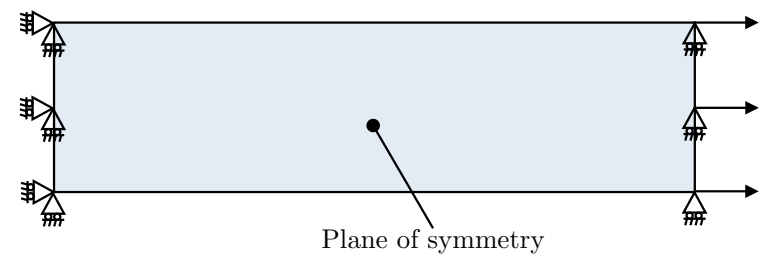

(b) Boundary conditions for the unnotched quasi-isotropic laminate

Figure 21: Finite element model of unnotched laminate under tension.

matrix cracks in Zone $\mathrm{C}$ clearly open up, and the $-45 / 0$ delamination in Zone $\mathrm{C}$ also develops significantly. The widespread delamination on the 90/-45 and -45/0 interfaces, together with the opened cracks in Zone $\mathrm{C}$, indicate that the laminate has disintegrated. The predicted number of $0^{\circ}$ splits is smaller than that observed in the experiment (Figure 1b). If the failure patterns of above $90 \%$ damaged (i.e., $d_{\mathrm{m}} \geq 0.9$ ) elements/SEs are plotted, then many more underlying $0^{\circ}$ splits can be revealed (Figure 24), the density and extent of which correspond closely with the experimental image in Figure 1b. In addition, the predicted delamination with $d_{\mathrm{m}} \geq 0.9$ in Figure 24 basically covers the entire area of the laminate, again suggesting that the laminate has completely disintegrated at final failure.

\section{Discussions}

When modelling delamination with cohesive elements, the cohesive zone (the zone where the interface is separating, but residual traction exists) must be modelled with a sufficient number of elements to ensure an accurate prediction of delamination $[8,11,13]$. In the above applications, meshes with elements of $0.5 \mathrm{~mm}$ to $1.0 \mathrm{~mm}$ in length are used. In generic situatoins, such meshes could be coarse for the accurate modelling of delamination using cohesive elements $[11,13]$. In the above applications, the delamination is mainly driven in Mode II and it occurs between thick ply-blocks, both of which contribute to increase the cohesive zone size [8]. As a result, the meshes used here are sufficient to capture the cohesive zones around the delamination fronts. Figure 25 shows the the damage index $\left(d_{\mathrm{m}}\right)$ of cohesive elements on the $-45 / 0$ interface of the two application cases. It is clear that there are many elements in the cohesive zone (where the elements are in the process of softening, i.e., $0<d_{\mathrm{m}}<1$ ) around the failed region (i.e., $d_{\mathrm{m}}=1$ ).

Figure 22c shows that the matrix crack density in certain regions has reached the maximum density allowed by the mesh, i.e., one matrix crack is propagating in every element. The maximum matrix crack density in the limit of mesh refinement is bounded by the strain energy available to propagate the cracks. 


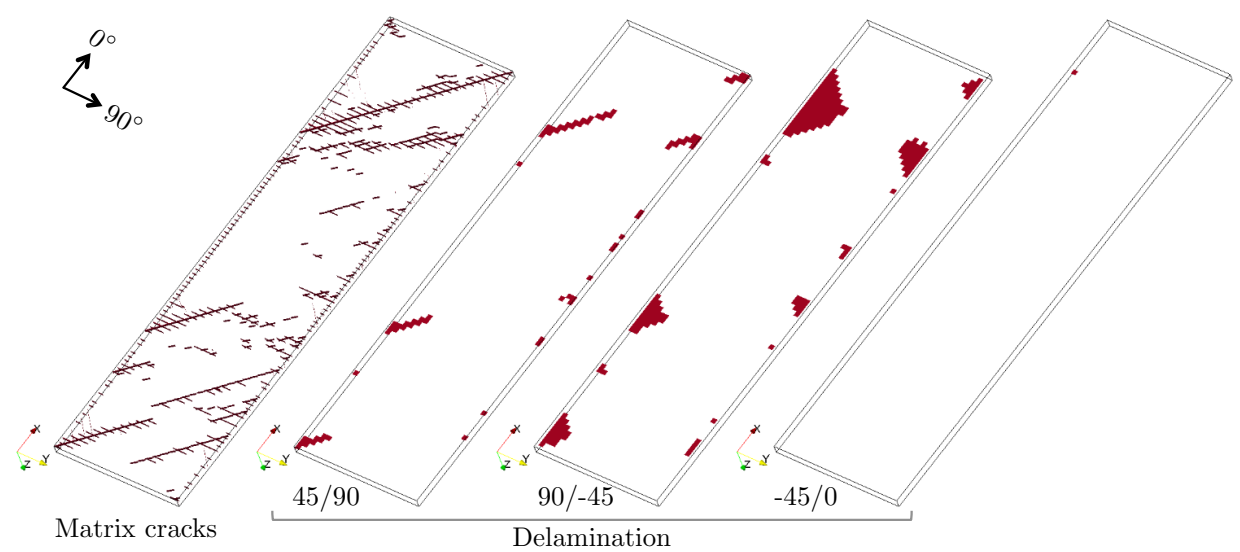

(a) Initial-stage failure patterns.

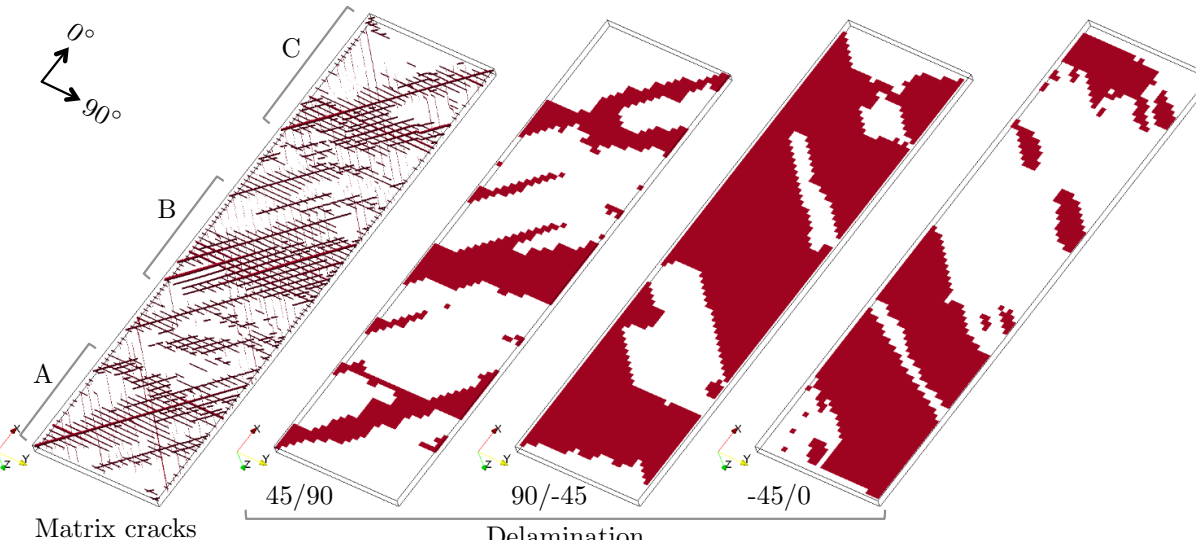

(b) Mid-stage failure patterns.

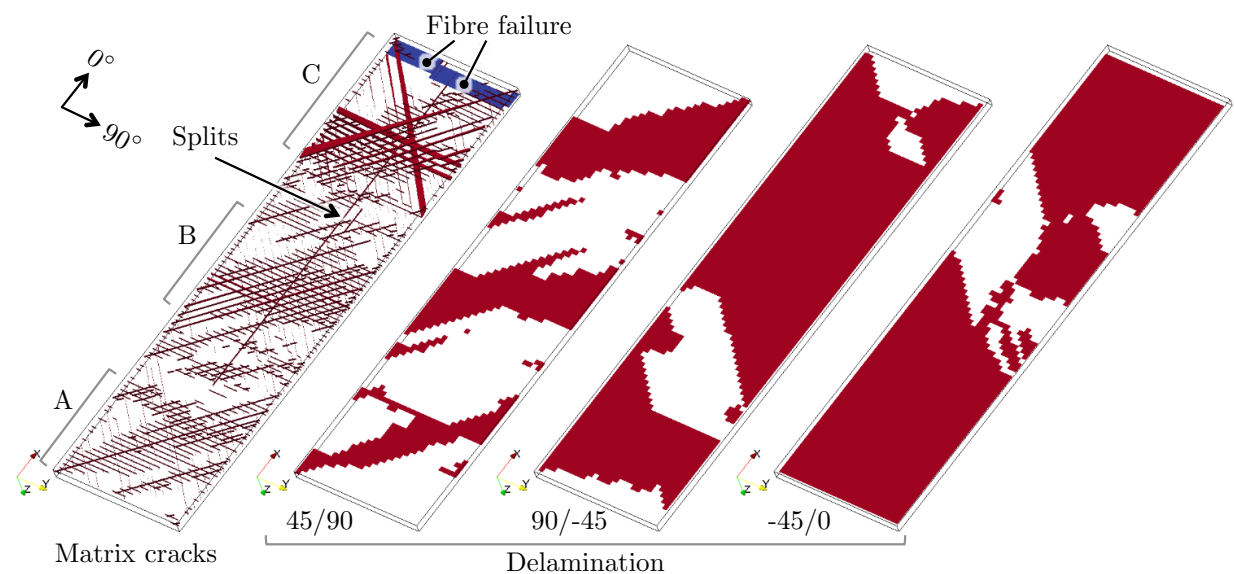

(c) Final failure patterns.

Figure 22: Failure pattern predictions of the unnotched $\left[45_{4} / 90_{4} /-45_{4} / 0_{4}\right]_{\mathrm{S}}$ laminate in [1]. 


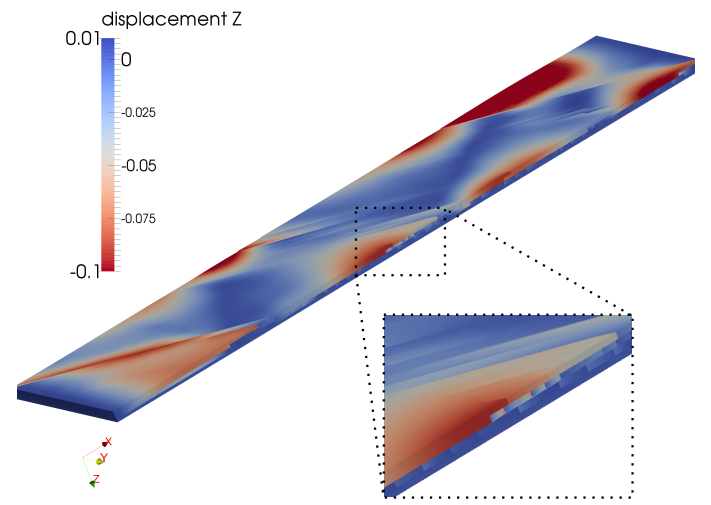

Figure 23: The predicted out-of-plane displacement contour shows the peel-off of the $45^{\circ}$ and $90^{\circ}$ plies from the edge.

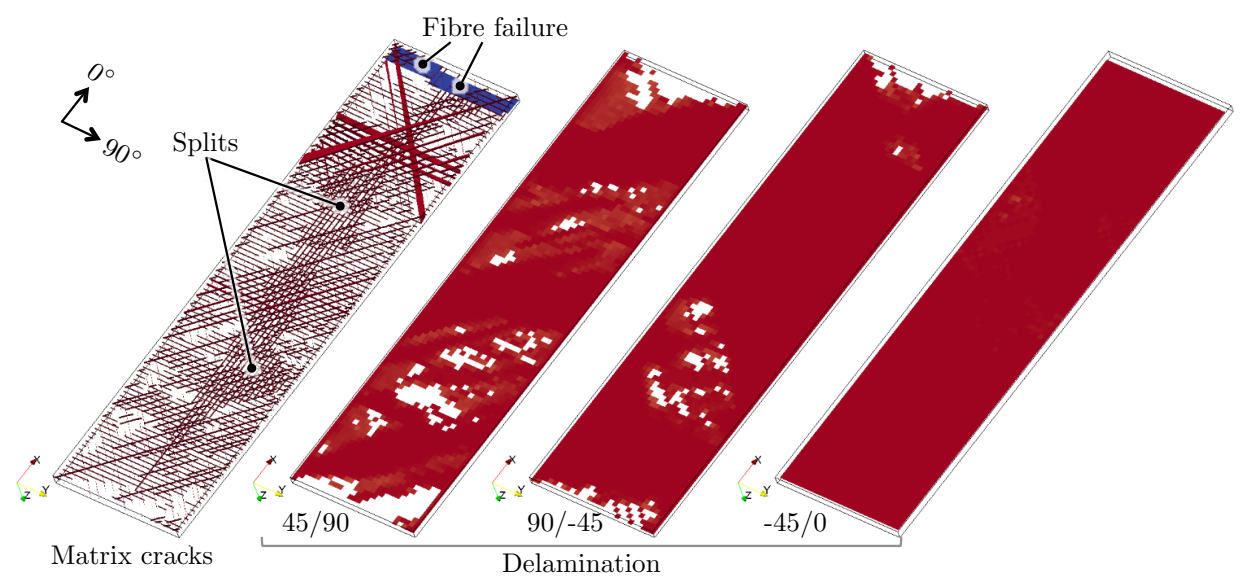

Figure 24: Final failure patterns of $d_{\mathrm{m}} \geq 0.9$.

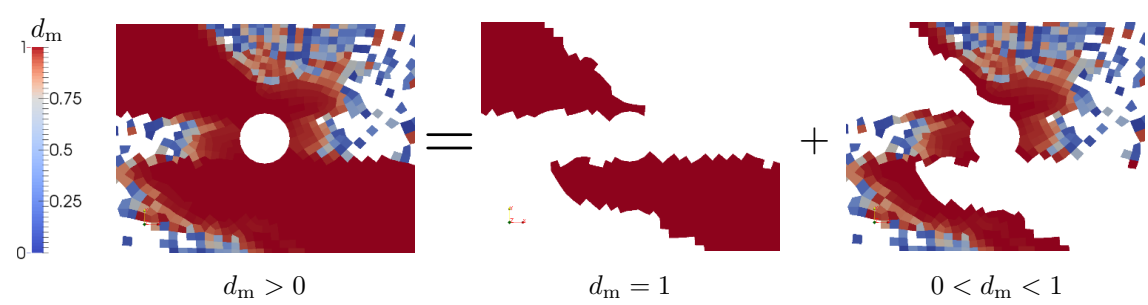

(a) Damage index of cohesive elements around the hole of the OHT model.
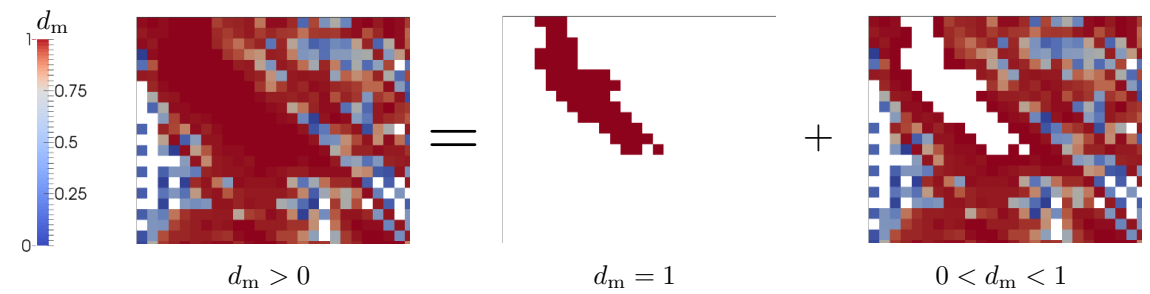

(b) Damage index of cohesive elements at a corner of the unnotched model.

Figure 25: Damage index of cohesive elements on the $-45 / 0$ interface of the two application examples. 
This density saturates, when the strain energy is insufficient to propagate a new matrix crack between two adjacent matrix cracks. In order to capture this saturation phenomenon, the mesh density must be several times larger than the saturation crack density to allow multiple elements between every two matrix cracks, such that the stress profile and strain energy between cracks can be accurately captured. The study of the saturation of matrix crack density has been reported for two-dimensional, cross-ply problems in [46]. In the current study, the mesh refinement is still insufficient to capture the physical saturation density. As a result, the predicted density of matrix cracks is given by the mesh density. Nevertheless, with the current level of mesh refinement, the predicted number of matrix cracks is already substantial, and the interplay between the matrix cracks and delamination and the progression towards final failure of the laminate can be captured. As the global response of a laminate is often not strongly affected by the development of matrix cracks, solely the under-prediction of matrix crack density itself would not significantly alter the predicted global response of the laminate. However, the exact moment of widespread delamination, which occur by the propagation and coalescence of local delamination induced my matrix cracks, could be delayed due to the larger distance between matrix cracks in modelling than in reality. A more refined mesh would be needed to capture accurately the matrix crack saturation density and the propagation and coalescence of local delamination.

For implicit analysis, it is often challenging to obtain convergence of the simulation, especially when a large number of cohesive cracks are being modelled. The list of Abaqus solver parameters used in this study is shown in Figure 26 in the appendix. Note that artificial viscosity is not used. In the authors' experience, the most critical parameter for reaching convergence is the maximum number of equilibrium iterations allowed for the Newton solver. If this number is insufficient with respect to the number of DoFs in the mesh, the solver would not be able to find the equilibrium for all the DoFs and would terminate the analysis prematurely. Therefore, this number should be increased together with the number of DoFs in the mesh.

In the application examples, matrix cracks and delamination could remove the initial stress concentration, resulting in a relatively uniform stress state in the 0 plies. However, the distributed splits and the clamping boundary condition at the grips introduce additional locations of stress concentration, which eventually lead to the onset of fibre failure in these locations (Figure 20b and Figure 22c). A large number of iterations are needed for the Newton method to find the eventual location of fibre failure out of competing regions of stress concentration, e.g., the two gripped ends of the unnotched laminate.

\section{Conclusions}

This paper presents the high-fidelity modelling of the progressive tensile failure of composites. In particular, the complex failure scenarios of the ply-blocked composite laminate, i.e., the high density of matrix cracks, the large area of delamination, the extensive matrix splittings and the distributed fibre breaking in the 0 plies, are targeted.

In order to accurately model the above-mentioned failure scenarios, a three-dimensional (3D), compositesoriented Floating Node Method (FNM) is developed. The definition of an element is enriched to include the connectivities of generic geometrical entities such as edges. Floating DoFs are allocated to these entities, such that they can represent the displacements of future-appearing nodes on the entities. An enriched ply element is constructed such that a cohesive matrix crack can be represented explicitly within its domain. An enriched cohesive element is formulated such that it takes into account of the matrix crack boundaries on the 
interface. A laminate element can be constructed such that all the plies and interfaces are modelled within the element, and a planar mesh can be used for arbitrary layups. The edge status variable approach facilitate the propagation of cracks in the mesh, such that a large number of cracks can be effectively propagated.

The 3D FNM is applied on the modelling of the progressive tensile failure of ply-blocked, quasi-isotropic laminates, where extensive matrix cracking, splitting and widespread delamination occur prior to the final fibre failure. It is shown that the proposed method accurately predicts the density and extent of matrix cracks and splits, the thereby induced delamination, the subsequent widespread propagation of the delamination which causes the disintegration of the laminate, and the final extensive splitting and distributed fibre breaking of the almost independent 0 plies. This complete process of progressive failure, up to the distributed fibre breaking of the loosened $0^{\circ}$ strips, is firstly reproduced by simulations. The results show that the 3D FNM is a powerful method for the high-fidelity modelling of progressive tensile failure of composites.

\section{Acknowledgement}

The authors would like to acknowledge the research grant (No. R265000463112) from the Ministry of Education of Singapore.

\section{References}

[1] M. R. Wisnom, B. Khan, S. R. Hallett, Size effects in unnotched tensile strength of unidirectional and quasi-isotropic carbon/epoxy composites, Composite Structures 84 (1) (2008) 21-28.

[2] B. G. Green, M. R. Wisnom, S. R. Hallett, An experimental investigation into the tensile strength scaling of notched composites, Composites Part A-Applied Science and Manufacturing 38 (3) (2007) 867-878.

[3] X. Xu, M. R. Wisnom, Y. Mahadik, S. R. Hallett, An experimental investigation into size effects in quasi-isotropic carbon/epoxy laminates with sharp and blunt notches, Composites Science and Technology 100 (2014) $220-227$.

[4] O. J. Nixon-Pearson, S. R. Hallett, An investigation into the damage development and residual strengths of open-hole specimens in fatigue, Composites Part A: Applied Science and Manufacturing 69 (2015) 266-278.

[5] H. H. Daken, J. W. Mar, Splitting initiation and propagation in notched unidirectional graphite/epoxy composites under tension-tension cyclic loading, Composite Structures 4 (2) (1985) 111-133.

[6] Y. Qiu, M. A. Crisfield, G. Alfano, An interface element formulation for the simulation of delamination with buckling, Engineering Fracture Mechanics 68 (16) (2001) 1755-1776.

[7] P. P. Camanho, C. G. Dávila, M. F. de Moura, Numerical simulation of mixed-mode progressive delamination in composite materials, Journal of Composite Materials 37 (16) (2003) 1415-1438.

[8] Q. D. Yang, B. Cox, Cohesive models for damage evolution in laminated composites, International Journal of Fracture $133(2)(2005)$ 107-137.

[9] G. Alfano, On the influence of the shape of the interface law on the application of cohesive-zone models, Composites Science and Technology 66 (6) (2006) 723-730.

[10] S. T. Pinho, L. Iannucci, P. Robinson, Formulation and implementation of decohesion elements in an explicit finite element code, Composites Part A-Applied Science and Manufacturing 37 (5) (2006) 778-789.

[11] A. Turon, C. G. Dávila, P. P. Camanho, J. Costa, An engineering solution for mesh size effects in the simulation of delamination using cohesive zone models, Engineering Fracture Mechanics 74 (10) (2007) 1665-1682.

[12] X. Li, S. R. Hallett, M. R. Wisnom, Predicting the effect of through-thickness compressive stress on delamination using interface elements, Composites Part A-Applied Science and Manufacturing 39 (2) (2008) 218-230.

[13] P. W. Harper, S. R. Hallett, Cohesive zone length in numerical simulations of composite delamination, Engineering Fracture Mechanics 75 (16) (2008) 4774-4792.

[14] S. R. Hallett, B. G. Green, W. G. Jiang, M. R. Wisnom, An experimental and numerical investigation into the damage mechanisms in notched composites, Composites Part A-Applied Science and Manufacturing 40 (5) (2009) 613-624. 
[15] A. Turon, P. P. Camanho, J. Costa, J. Renart, Accurate simulation of delamination growth under mixed-mode loading using cohesive elements: Definition of interlaminar strengths and elastic stiffness, Composite Structures 92 (8) (2010) 1857-1864.

[16] M. R. Wisnom, Modelling discrete failures in composites with interface elements, Composites Part A-Applied Science and Manufacturing 41 (7) (2010) 795-805.

[17] M. Ridha, V. B. C. Tan, T. E. Tay, Traction-separation laws for progressive failure of bonded scarf repair of composite panel, Composite Structures 93 (4) (2011) 1239-1245.

[18] R. de Borst, Numerical aspects of cohesive-zone models, Engineering Fracture Mechanics 70 (14) (2003) $1743-1757$.

[19] G. Liu, K. Tang, H. Bao, Failure analysis of notched fiber-reinforced composite materials, in: the 20th International Conference on Composite Materials, Copenhagen, 19-24 July, 2015.

[20] S. T. Pinho, L. Iannucci, P. Robinson, Physically based failure models and criteria for laminated fibre-reinforced composites with emphasis on fibre kinking. Part II: FE implementation, Composites Part A-Applied Science and Manufacturing 37 (5) (2006) 766-777.

[21] P. P. Camanho, P. Maimi, C. G. Dávila, Prediction of size effects in notched laminates using continuum damage mechanics, Composites Science and Technology 67 (13) (2007) 2715-2727.

$[22]$ B. Y. Chen, T. E. Tay, P. M. Baiz, S. T. Pinho, Numerical analysis of size effects on open-hole tensile composite laminates, Composites Part A: Applied Science and Manufacturing 47 (0) (2013) 52 - 62.

[23] S. T. Pinho, G. M. Vyas, P. Robinson, Material and structural response of polymer-matrix fibre-reinforced composites: Part B, Journal of Composite Materials 47 (6-7) (2013) 679-696.

[24] U. Mandel, R. Taubert, R. Hinterhölzl, Mechanism based nonlinear constitutive model for composite laminates subjected to large deformations, Composite Structures 132 (2015) 98-108.

[25] F. P. van der Meer, L. J. Sluys, Continuum Models for the Analysis of Progressive Failure in Composite Laminates, Journal of Composite Materials 43 (20) (2009) 2131-2156.

[26] K. Song, Y. Li, C. A. Rose, Continuum Damage Mechanics Models for the Analysis of Progressive Failure in Open-Hole Tension Laminates, AIAA 1861 (2011) 1-18.

[27] T. Belytschko, T. Black, Elastic crack growth in finite elements with minimal remeshing, International Journal for Numerical Methods in Engineering 45 (5) (1999) 601-620.

[28] N. Moës, J. Dolbow, T. Belytschko, A finite element method for crack growth without remeshing, International Journal for Numerical Methods in Engineering 46 (1) (1999) 131-150.

[29] G. N. Wells, L. J. Sluys, A new method for modelling cohesive cracks using finite elements, International Journal for Numerical Methods in Engineering 50 (12) (2001) 2667-2682.

[30] N. Sukumar, Z. Y. Huang, J. H. Prevost, Z. Suo, Partition of unity enrichment for bimaterial interface cracks, International Journal for Numerical Methods in Engineering 59 (8) (2004) 1075-1102.

[31] X. S. Sun, V. B. C. Tan, G. Liu, T. E. Tay, An enriched element-failure method (REFM) for delamination analysis of composite structures, International Journal for Numerical Methods in Engineering 79 (6) (2009) 639-666.

[32] T. Nagashima, H. Suemasu, X-FEM analyses of a thin-walled composite shell structure with a delamination, Computers \& Structures 88 (9-10) (2010) 549-557.

[33] S. E. Ashari, S. Mohammadi, Delamination analysis of composites by new orthotropic bimaterial extended finite element method, International Journal for Numerical Methods in Engineering 86 (13) (2011) 1507-1543.

[34] E. V. Iarve, M. R. Gurvich, D. H. Mollenhauer, C. A. Rose, C. G. Dávila, Mesh-independent matrix cracking and delamination modeling in laminated composites, International Journal for Numerical Methods in Engineering 88 (8) (2011) 749-773.

[35] M. J. Swindeman, E. V. Iarve, R. A. Brockman, D. H. Mollenhauer, S. R. Hallett, Strength Prediction in Open Hole Composite Laminates by Using Discrete Damage Modeling, AIAA Journal 51 (2013) 936-945.

[36] T. E. Tay, X. S. Sun, V. B. C. Tan, Recent efforts toward modeling interactions of matrix cracks and delaminations: an integrated XFEM-CE approach, Advanced Composite Materials 23 (5-6) (2014) 391-408.

[37] A. Hansbo, P. Hansbo, A finite element method for the simulation of strong and weak discontinuities in solid mechanics, Computer Methods in Applied Mechanics and Engineering 193 (33-35) (2004) 3523-3540.

[38] P. M. A. Areias, J. H. Song, T. Belytschko, Analysis of fracture in thin shells by overlapping paired elements, Computer Methods in Applied Mechanics and Engineering 195 (41-43) (2006) 5343-5360.

[39] J.-H. Song, P. M. A. Areias, T. Belytschko, A method for dynamic crack and shear band propagation with phantom nodes, International Journal for Numerical Methods in Engineering 67 (6) (2006) 868-893. 
[40] F. P. van der Meer, L. J. Sluys, A phantom node formulation with mixed mode cohesive law for splitting in laminates, International Journal of Fracture 158 (2) (2009) 107-124.

[41] F. P. van der Meer, L. J. Sluys, S. R. Hallett, M. R. Wisnom, Computational modeling of complex failure mechanisms in laminates, Journal of Composite Materials 46 (5) (2012) 603-623.

[42] F. P. van der Meer, C. G. Dávila, Cohesive modeling of transverse cracking in laminates under in-plane loading with a single layer of elements per ply, International Journal of Solids and Structures 50 (20-21) (2013) $3308-3318$.

[43] D. S. Ling, Q. D. Yang, B. Cox, An augmented finite element method for modeling arbitrary discontinuities in composite materials, International Journal of Fracture 156 (1) (2009) 53-73.

[44] X. J. Fang, Z. Q. Zhou, B. N. Cox, Q. D. Yang, High-fidelity simulations of multiple fracture processes in a laminated composite in tension, Journal of the Mechanics and Physics of Solids 59 (7) (2011) 1355-1373.

[45] X. J. Fang, Q. D. Yang, B. N. Cox, Z. Q. Zhou, An augmented cohesive zone element for arbitrary crack coalescence and bifurcation in heterogeneous materials, International Journal for Numerical Methods in Engineering 88 (2011) 841-861.

[46] B. Y. Chen, S. T. Pinho, N. V. D. Carvalho, P. M. Baiz, T. E. Tay, A floating node method for the modelling of discontinuities in composites, Engineering Fracture Mechanics 127 (2014) $104-134$.

[47] N. Moës, E. Béchet, M. Tourbier, Imposing Dirichlet boundary conditions in the extended finite element method, International Journal for Numerical Methods in Engineering 67 (12) (2006) 1641-1669.

[48] T.-P. Fries, T. Belytschko, The extended/generalized finite element method: An overview of the method and its applications, International Journal for Numerical Methods in Engineering 84 (3) (2010) 253-304.

[49] N. V. De Carvalho, B. Y. Chen, S. T. Pinho, J. G. Ratcliffe, P. M. Baiz, T. E. Tay, Modeling delamination migration in cross-ply tape laminates, Composites Part A: Applied Science and Manufacturing 71 (2015) 192-203.

[50] M. J. Laffan, S. T. Pinho, P. Robinson, L. Iannucci, Measurement of the in situ ply fracture toughness associated with mode I fibre tensile failure in FRP. Part II: Size and lay-up effects, Composites Science and Technology 70 (4) (2010) $\underline{614-621 .}$

[51] G. Liu, K. Dai, T. Nguyen, A smoothed finite element method for mechanics problems, Computational Mechanics 39 (6) (2007) 859-877. 
TIME INCREMENTATION CONTROL PARAMETERS:

*** FIRST EQUILIBRIUM ITERATION FOR CONSECUTIVE DIVERGENCE CHECK 800

*** EQUILIBRIUM ITERATION AT WHICH LOG. CONVERGENCE RATE CHECK BEGINS 1000 EQUILIBRIUM ITERATION AFTER WHICH ALTERNATE RESIDUAL IS USED

$\star \star \star *$ MAXIMUM EQUILIBRIUM ITERATIONS ALLOWED 1500

$\star * *$ EQUILIBRIUM ITERATION COUNT FOR CUT-BACK IN NEXT INCREMENT 1200

*** MAXIMUM EQUILIB. ITERS IN TWO INCREMENTS FOR TIME INCREMENT INCREASE 50 MAXIMUM ITERATIONS FOR SEVERE DISCONTINUITIES

*** MAXIMUM CUT-BACKS ALLOWED IN AN INCREMENT 125

MAXIMUM DISCON. ITERS IN TWO INCREMENTS FOR TIME INCREMENT INCREASE 50

MAXIMUM CONTACT AUGMENTATIONS FOR *SURFACE BEHAVIOR, AUGMENTED LAGRANGE 50

$\begin{array}{ll}\text { CUT-BACK FACTOR AFTER DIVERGENCE } & 0.2500\end{array}$

CUT-BACK FACTOR FOR TOO SLOW CONVERGENCE $\quad 0.5000$

CUT-BACK FACTOR AFTER TOO MANY EQUILIBRIUM ITERATIONS $\quad 0.7500$

CUT-BACK FACTOR AFTER TOO MANY SEVERE DISCONTINUITY ITERATIONS 0.2500

CUT-BACK FACTOR AFTER PROBLEMS IN ELEMENT ASSEMBLY $\quad 0.2500$

INCREASE FACTOR AFTER TWO INCREMENTS THAT CONVERGE QUICKLY 1.500

MAX. TIME INCREMENT INCREASE FACTOR ALLOWED 1.500

MAX. TIME INCREMENT INCREASE FACTOR ALLOWED (DYNAMICS) 1.250

MAX. TIME INCREMENT INCREASE FACTOR ALLOWED (DIFFUSION) 2.000

MINIMUM TIME INCREMENT RATIO FOR EXTRAPOLATION TO OCCUR 0.1000

MAX. RATIO OF TIME INCREMENT TO STABILITY LIMIT 1.000

FRACTION OF STABILITY LIMIT FOR NEW TIME INCREMENT $\quad 0.9500$

TIME INCREMENT INCREASE FACTOR BEFORE A TIME POINT 1.000

*** INDICATES USE OF NON-DEFAULT TIME INCREMENTATION CONTROL PARAMETER SELECTED WITH *CONTROLS

GLOBAL STABILIZATION CONTROL IS NOT USED

Figure 26: Abaqus solver parameters used in the simulation. 